\title{
A Numerical Study on the Oscillating Flow Induced by an Acoustic Field around Coal Particles
}

\author{
Genshan Jiang, ${ }^{1}$ Weilong $\mathrm{Xu}^{2}{ }^{2}$ Yuechao Liu, ${ }^{2}$ Yapan $\mathrm{Wu},{ }^{1}$ and Qian Kong ${ }^{1}$ \\ ${ }^{1}$ North China Electric Power University, Baoding 071003, China \\ ${ }^{2}$ North China Electric Power University, Beijing 102206, China
}

Correspondence should be addressed to Weilong Xu; xuweilong@ncepu.edu.cn

Received 25 May 2016; Accepted 26 July 2016

Academic Editor: Xiang Wang

Copyright (c) 2016 Genshan Jiang et al. This is an open access article distributed under the Creative Commons Attribution License, which permits unrestricted use, distribution, and reproduction in any medium, provided the original work is properly cited.

In order to investigate the acoustically driven oscillating flow around coal particles in the power plant boiler, the two-dimensional, unsteady mass and momentum conservation equations for laminar flow in spherical coordinates are developed numerically. The velocity field, axial pressure gradient, shear stress, and flow separation angle on the particle surface are carefully analyzed with different values of acoustic Reynolds number and Strouhal number. The minimum frequency required for flow separation is also investigated with different SPL (sound pressure level). The axial pressure gradient, shear stress, and separation angle on the surface are proportional to the magnitude of the oscillating flow velocity at low frequency $(\sim 50 \mathrm{~Hz})$. However, those physical quantities have different values at high frequency $(\sim 5000 \mathrm{~Hz})$, due to the combined effect of curvature and the flow acceleration.

\section{Introduction}

Over the years, considerable researches have been carried out on oscillating flows over bodies of various shapes because of the related engineering application [1]. The rates of heat and mass transfer from the sphere are enhanced by the oscillation of the surrounding fluid. This phenomenon has good potential in some areas, such as the pulse combustion [2], food refrigeration [3], and heat exchanger $[4,5]$.

The theoretical study of oscillating flow over sphere dates back to the work of Bassett [6]. An experiment had been conducted by Odar and Hamilton [7] on oscillating flow over a sphere in attempt to modify Bassett's solution. Mei [8] proposed a general dynamic equation including the quasisteady drag, history force, and added-mass force in the time domain for particle motions at finite Reynolds number (order of several hundred). It was found that the form of history force obtained performed consistently better than that of Odar and Hamilton. Chang and Maxey [9] investigated the oscillating flow over a sphere with the frequency up to $10 \mathrm{~Hz}$ and the Reynolds numbers up to 16.7, by using the numerical calculation method. They found that, at very low Reynolds number, separation took place during the deceleration period and there was no separation during the acceleration period.
Then, Alassar [10] extended the Reynolds number to 200 with the series truncation method and had careful analyses on the separation angle and the wake length. Alassar [10] also investigated the phenomenon of second motion (acoustic streaming) created by the oscillating flow over a sphere, by solving the full Navier-Stokes equations. However, all the researches above on oscillating flow over a sphere have one thing in common that the maximum excursion of sphere over one period of the flow is small, compared to the characteristic size of the particles.

When an acoustic field is applied to enhance the combustion and the heat and mass transfer to and from coal particles, the maximum excursion is large compared to the characteristic size of the coal particles. In this case, Pozrikidis [11] investigated the problem of viscous oscillating flow over a particle at low Reynolds numbers (order of ten), by solving the unsteady stokes equation with boundary-integral method. Ha [12] studied the oscillating flow driven by highintensity acoustic field over a spherical particle (the diameter is $100 \mu \mathrm{m}$ ) in the air. Some issues were discussed as well, such as the flow structure, axial pressure gradient, shear stress, and flow separation on the particle surface. Oscillating flow driven by a standing wave around a solid particle (the diameter is at the nanoscale) was investigated by Sadhal [13]. 


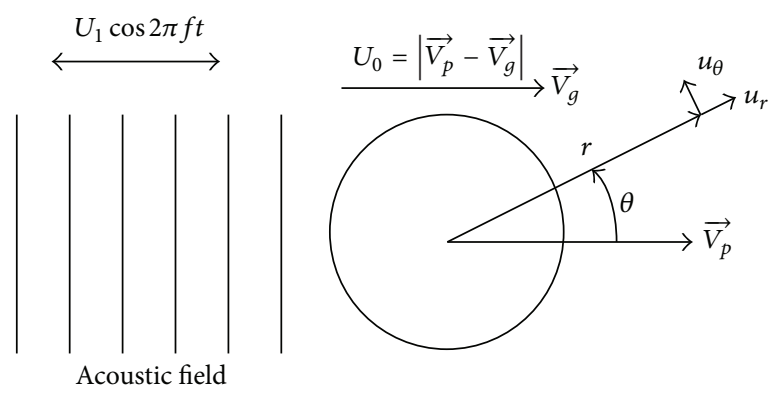

FIGURE 1: Schematic diagram showing the geometry and some of the nomenclature used to simulate oscillating flows over a spherical particle in the presence of an acoustic field.

TABLE 1: Source terms $S_{\phi}$ in (1)

\begin{tabular}{ll}
\hline$\quad S_{\phi}$ & $-\frac{\partial P}{\partial r}+\frac{1}{r^{2}} \frac{\partial}{\partial r}\left(\mu r^{2} \frac{\partial u_{r}}{\partial r}\right)+\frac{1}{r \sin \theta} \frac{\partial}{\partial \theta}\left(\mu \sin \theta \frac{\partial u_{\theta}}{\partial r}\right)$ \\
$u_{r} \quad-\frac{1}{r^{2} \sin \theta} \frac{\partial}{\partial r}\left(\mu \sin \theta u_{\theta}\right)-\frac{2 \mu}{r^{2}} \frac{\partial u_{\theta}}{\partial \theta}-4 \mu \frac{u_{r}}{r^{2}}-2 \mu \frac{u_{\theta} \cot \theta}{r^{2}}+\rho \frac{u_{\theta}}{r^{2}}$ \\
& $\frac{1}{r} \frac{\partial P}{\partial \theta}+\frac{1}{r^{2}} \frac{\partial}{\partial r}\left(\mu r^{2} \frac{\partial u_{\theta}}{\partial r}\right)+\frac{1}{r^{2} \sin \theta} \frac{\partial}{\partial \theta}\left(\mu \sin \theta \frac{\partial u_{\theta}}{\partial \theta}\right)$ \\
& $+\frac{2}{r \sin \theta} \frac{\partial}{\partial \theta}\left(\mu \sin \theta \frac{u_{r}}{r}\right)+\frac{u}{r} \frac{\partial u_{\theta}}{\partial r}+\frac{\mu}{r^{2}} \frac{\partial u_{r}}{\partial \theta}-\mu \frac{u_{\theta}}{r^{2}}$ \\
& $-2 \mu \frac{u_{r} \cot \theta}{r^{2}}-2 \mu \frac{u_{\theta} \cot ^{2} \theta}{r^{2}}-\frac{1}{r^{2}} \frac{\partial}{\partial r}\left(\mu r u_{\theta}\right)-\rho \frac{u_{r} u_{\theta}}{r^{2}}$
\end{tabular}

In this paper, the two-dimensional, unsteady mass and momentum conservation equations for laminar flow are solved for the oscillating flow induced by an acoustic field around coal particles. The parameters of flue gas in the real power plant boiler are also taken into account. The flow velocity, axial pressure gradient, shear stress, and flow separation on the coal particle surface are analyzed in different Reynolds numbers and Strouhal numbers. Although there is not much about the mechanism of acoustic enhancement of heat and mass transfer from coal particles, this study highlights its good application prospect in the power plant boiler.

\section{The Model}

2.1. Governing Equation and Physical Model. The general differential equations can be written as [14]

$$
\begin{gathered}
\frac{\partial}{\partial t}(\rho \phi)+\frac{1}{r^{2}} \frac{\partial}{\partial r}\left(r^{2} \rho u_{r} \phi\right)+\frac{1}{r \sin \theta} \frac{\partial}{\partial \theta}\left(\sin \theta \rho u_{\theta} \phi\right) \\
=\frac{1}{r^{2}} \frac{\partial}{\partial r}\left(\mu r^{2} \frac{\partial \phi}{\partial r}\right)+\frac{1}{r^{2} \sin \theta} \frac{\partial}{\partial \theta}\left(\mu \sin \theta \frac{\partial \phi}{\partial \theta}\right)+S_{\phi}
\end{gathered}
$$

where $u_{r}, u_{\theta}$ are the radial velocity and tangential velocity, respectively. $S_{\phi}$ is the source term and its expression in the radial is different from that in tangential directions, as shown in Table 1.

Figure 1 shows the acoustic field and the particle geometry with some nomenclature. A circumferential symmetry is assumed around an axis which passes through the center of the particle and is parallel to the flow direction.

2.2. Boundary Conditions. The governing equation (1) has the following initial and boundary conditions.

Initial Conditions (at $t=0)$. Consider

$$
\phi=\phi_{0} \text {. }
$$

Boundary Conditions (When $t>0$ ). Consider the following: at $\theta=0$ and $\pi$ (symmetry conditions):

$$
\frac{\partial \phi}{\partial \theta}=0
$$

at $r=R$

$$
\phi=\phi_{p}
$$

and as $r \rightarrow \infty$,

$$
\begin{aligned}
& u_{\theta}=-U_{1} \cos (2 \pi f t) \sin \theta \\
& u_{r}=U_{1} \cos (2 \pi f t) \cos \theta
\end{aligned}
$$

In (4), the subscript $p$ in $\phi_{p}$ represents the particle surface. As the boundary conditions constrain to no slip and impermeability, $u_{r p}$ and $u_{\theta p}$ at the particle surface are both zero in (4). In a traveling wave acoustic field with the sound pressure $P_{r m s}$ (the unit is $\mathrm{N} / \mathrm{m}^{2}$ ) and the acoustic impedance $\rho_{g} C_{0}$ ( $\rho_{g}$ is the flus gas density, $C_{0}$ is the sound speed in boiler), the peak value of acoustic velocity $U_{1}$ can be calculated by

$$
U_{1}=\frac{\sqrt{2} P_{r m s}}{\rho_{g} C_{0}},
$$

where $P_{r m s}=10^{A}$ and $A=\left(L_{P}-94\right) / 20, L_{P}$ represents the sound pressure level, and the unit is $\mathrm{dB}$.

In most of power plant boilers, the furnace is in the state under negative pressure and high temperature. In this paper, the pressure $P_{0}$ is fixed at the value of $101225 \mathrm{~Pa}$ (negative pressure $100 \mathrm{~Pa}$ ) and temperature $T_{0}$ at $1200^{\circ} \mathrm{C}$. The thermophysical properties of flue gas are listed in Table 2. Based on the basic theory of sound wave, the sound speed in boiler could be calculated by $C_{0}=\sqrt{\gamma P_{0} / \rho_{g}}(\gamma$ is specific heat ratio, $\gamma=1.36$ ); then it can be known that $C_{0} \approx 757.37 \mathrm{~m} / \mathrm{s}$ in boiler. 
TABLE 2: Thermal physical properties of flue gas under furnace pressure.

\begin{tabular}{ccccccc}
\hline$t /{ }^{\circ} \mathrm{C}$ & $\rho / \mathrm{kg} \cdot \mathrm{m}^{-3}$ & $C_{p} / \mathrm{kJ} \cdot \mathrm{kg}^{-1} \cdot \mathrm{K}^{-1}$ & $\lambda \times 10^{2} / \mathrm{W} \cdot \mathrm{m}^{-1} \cdot \mathrm{K}^{-1}$ & $a \times 10^{6} / \mathrm{m}^{2} \cdot \mathrm{s}^{-1}$ & $\mu \times 10^{6} / \mathrm{kg} \cdot \mathrm{m}^{-1} \cdot \mathrm{s}^{-1}$ & $\nu \times 10^{6} / \mathrm{m}^{2} \cdot \mathrm{s}^{-1}$ \\
\hline 1200 & 0.240 & 1.340 & 12.62 & 392.4 & 53.0 & 221.0 \\
\hline
\end{tabular}

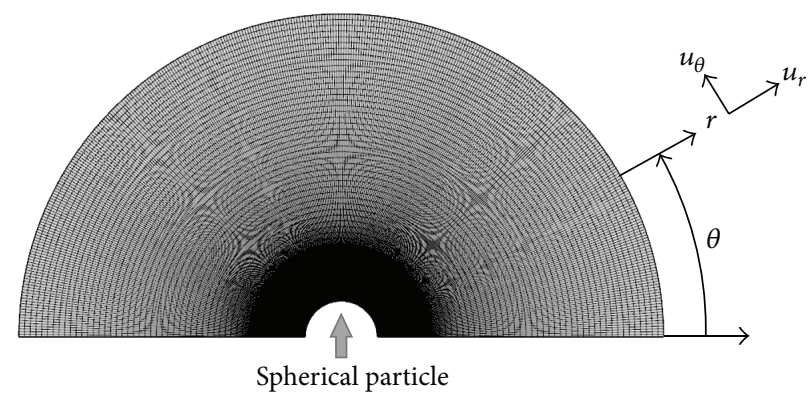

FIgURE 2: Calculation model and computational grid.

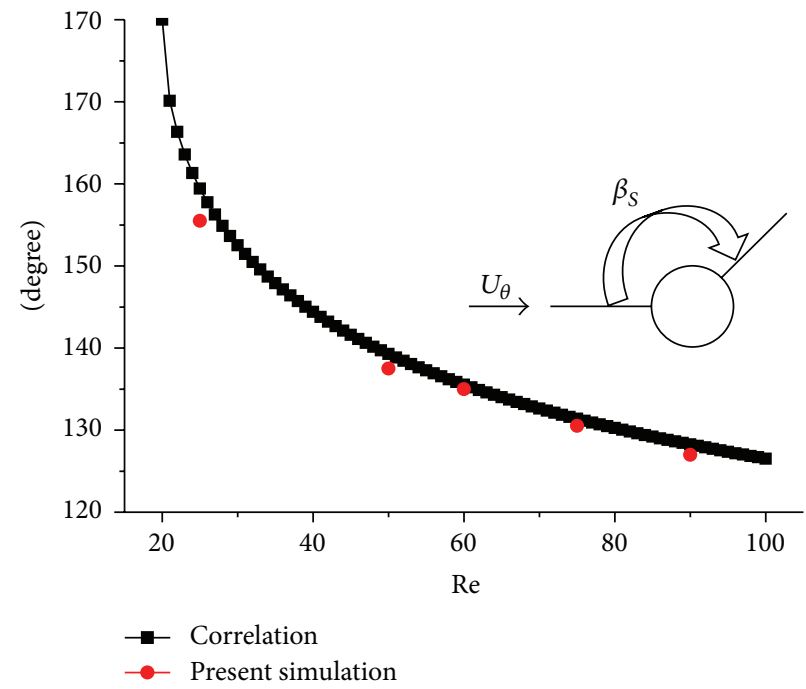

FIgURE 3: Comparison of separation angle $\beta$ obtained from previous correlation and the present simulation.

2.3. Meshing and Convergence Criteria. In the present simulation, the particle diameter is fixed at $100 \mu \mathrm{m}$ which is average size of pulverized coal particles in power plant. The numerical solution domain is chosen as 10 times the particle diameter and is meshed with 360 grids points in the axial direction and 50 in the radial direction. The calculation model is shown in Figure 2.

The conservation equations given by (1) are solved, using a finite difference scheme. The numerical solution algorithm is the SIMPLEC. One period is divided evenly into 40 time intervals, so the numerical time step is $t=1 / 40 \mathrm{f}$. During the SIMPLEC iteration procedure, the convergence criteria $\varepsilon_{\phi}$ are defined as follows:

$$
\varepsilon_{\phi}=\sum\left|\frac{\phi_{\text {new }}-\phi_{\text {old }}}{\phi_{\text {new }}}\right|
$$

where $\phi_{\text {old }}, \phi_{\text {new }}$ represent the values of $u_{r}$ and $u_{\theta}$ during the previous iteration and that during the present iteration, respectively. $\varepsilon_{\phi}$ is set to 0.005 in this paper.
2.4. The Accuracy of the Calculation Model Validation. It is well known that steady flow over a solid sphere separates at a Reynolds number (Re) of 20 (based on diameter) and forms a steady axisymmetric wake behind the body. With the increasing $\mathrm{Re}$, this added recirculating zone grows in size but remains essentially steady and axisymmetric up to 212 approximately. When Re is beyond 270, the flow behind the sphere undergoes yet another transition and develops into a fully three-dimensional, time dependent wake with periodic vortex shedding. When Re is between 420 and 800, the flow starts a transition with vortex shedding irregularly [15]. In this case by using empirical correlation for the steady Reynolds number range of 10 to 400 , the separation angle from the front stagnation point can be expressed as [16]

$$
\beta_{s}=180-42.5\left[\operatorname{In}\left(\frac{\mathrm{Re}_{0}}{20}\right)\right]^{0.483},
$$

where $\operatorname{Re}_{0}$ is the steady Reynolds number; $\operatorname{Re}_{0}=U_{0} D / \nu$ and $U_{0}$ is the steady slip velocity. Figure 3 shows the comparison of separation angle obtained from the correlation with the 


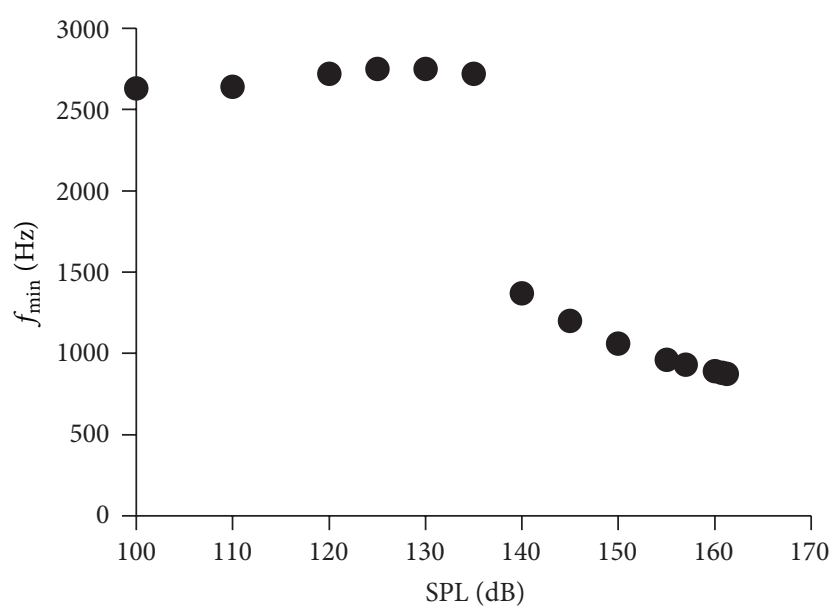

FIGURE 4: The minimum frequency required for flow separation in different SPL.

present simulation results. The simulation results are in good accordance with the results from the correlations.

\section{Results and Discussion}

The wall shear stress on the particle surface in spherical coordinate system could be calculated from the velocity profile as follows:

$$
\tau_{w}=\mu\left[r \frac{\partial}{\partial r}\left(\frac{u_{\theta}}{r}\right)+\frac{1}{r} \frac{\partial u_{r}}{\partial \theta}\right]_{r=R} .
$$

At the separation point, the wall shear stress $\tau_{w}$ is zero. After the simulation is ran for the case of an oscillating flow around a stationary spherical particle with different acoustic Reynolds numbers $\left(\operatorname{Re}_{1}, \operatorname{Re}_{1}=U_{1} D / \nu, U_{1}\right.$ is the amplitude of acoustic velocity, $D$ is the particle diameter, and $v$ is the flus gas kinematic viscosity) and Strouhal numbers (St, St = $f D / U_{1} ; f$ is the sound frequency) under the condition that $\mathrm{Re}_{0}=0.0$. The simulation would reach quasisteady state after several cycles. The following discussion concentrates on the results over one cycle right after a quasisteady state, unless it is mentioned otherwise.

3.1. The Minimum Frequency Required for Flow Separation in Different SPL. It is found that flow separation only happens when the frequency is not less than a certain value. As shown in Figure 4, the values of the minimum frequency required for flow separation varies slightly at first and then decreases with the increasing SPL. However, when SPL is over $162 \mathrm{~dB}$, flow separation could be observed in the range of audible frequencies. Therefore, the sound field with $\mathrm{SPL}=135 \mathrm{~dB}\left(U_{1}=\right.$ $0.875 \mathrm{~m} / \mathrm{s}), 167 \mathrm{~dB}\left(U_{1}=34.8 \mathrm{~m} / \mathrm{s}\right)$ and $f=50 \mathrm{~Hz}, 5000 \mathrm{~Hz}$ has been analyzed in this paper.

3.2. Flow Characteristics Induced by Acoustic Field with $S P L=$ $135 \mathrm{~dB}$. The oscillating flow induced by an acoustic field with SPL $=135 \mathrm{~dB}$ around coal particles is discussed in the following paragraph.
Figure 5 shows the velocity vectors in the domain for $\mathrm{Re}_{0}=$ 0.0 and $\mathrm{Re}_{1}=0.396(\mathrm{SPL}=135 \mathrm{~dB})$ corresponding to $f=$ $50 \mathrm{~Hz}(\mathrm{St}=0.00571)$ and $f=5000 \mathrm{~Hz}(\mathrm{St}=0.571)$. Flow separation (or wake) cannot be observed when $f=50 \mathrm{~Hz}$ ( $\mathrm{St}=$ 0.00571), as shown in Figure 5(a). However, if the frequency increases to $5000 \mathrm{~Hz}(\mathrm{St}=0.571)$, the flow separation and formation of a wake can be observed even if $\mathrm{Re}_{1}$ is less than 20, as shown in Figure 5(b). Regarding the unsteady flow, its flow field, separation angle, pressure distribution, and shear stress on the sphere surface are affected by not only the curvature but also the flow acceleration.

The normalized oscillating flow velocity $\left(U / U_{1}\right)$ and the acceleration $\left(\left(1 / 2 \pi f U_{1}\right)(d U / d t)\right)$ are shown in Figure 6. $\tau=$ $f \cdot t$ represents the dimensionless time. During $\tau=0.0 \sim 0.25$ and $\tau=0.75 \sim 1.0, U / U_{1}>0$, which means that the direction is from left to right. The direction is the opposite while $\tau=0.25 \sim$ 0.75 as $U / U_{1}<0$. The axial pressure gradient $((1 / r)(\partial P / \partial \theta))$, due to the flow acceleration in the potential flow, is expressed as

$$
\left(\frac{1}{r} \frac{\partial P}{\partial \theta}\right)_{\infty}=-2 \pi f \rho U_{1} \sin (2 \pi f t) \sin \theta
$$

A new angle $\beta$ related to the angle $\theta$ shown in Figure 1 is defined and it is measured from the front stagnation point (streamwise direction). Then during $\tau=0.0 \sim 0.25$ and $\tau=$ $0.75 \sim 1.0$,

$$
\beta=\pi-\theta
$$

during $\tau=0.25 \sim 0.75$,

$$
\beta=\theta \text {. }
$$

By substituting $\theta$ into (10) with (11) and (12), during $\tau=$ $0 \sim 0.25$ and $\tau=0.75 \sim 1.0$,

$$
\left(\frac{1}{r} \frac{\partial P}{\partial \beta}\right)_{\infty}=2 \pi f \rho U_{1} \sin (2 \pi f t) \sin \beta ;
$$

during $\tau=0.25 \sim 0.75$,

$$
\left(\frac{1}{r} \frac{\partial P}{\partial \beta}\right)_{\infty}=-2 \pi f \rho U_{1} \sin (2 \pi f t) \sin \beta
$$




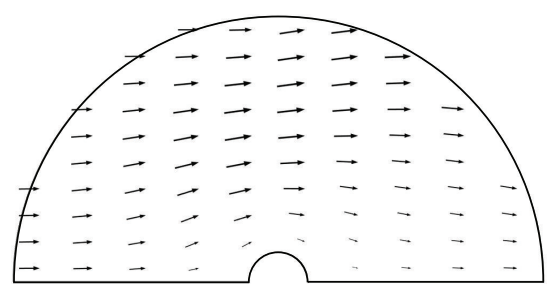

(1)

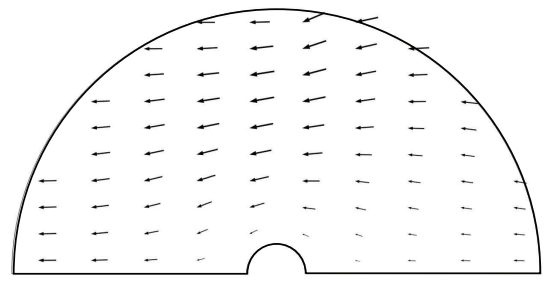

(4)

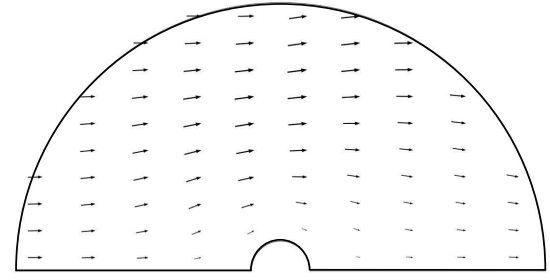

(2)

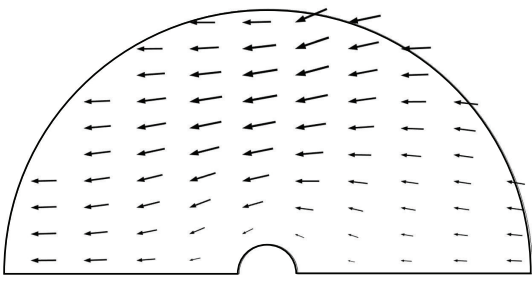

(5)

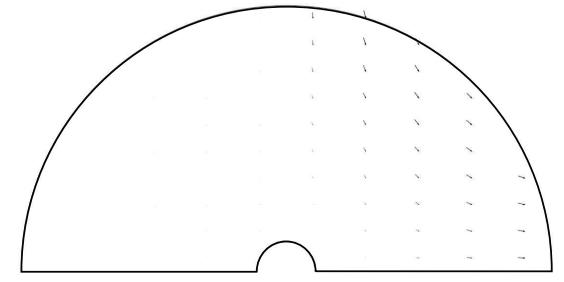

(3)

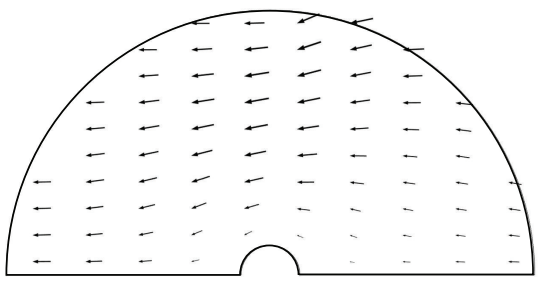

(6)

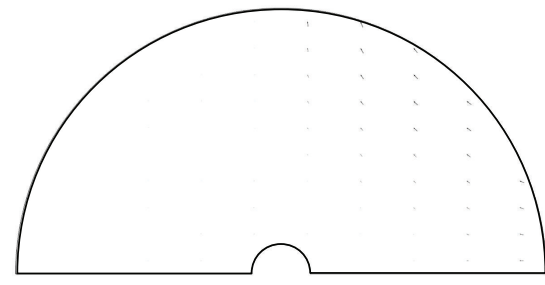

(7)

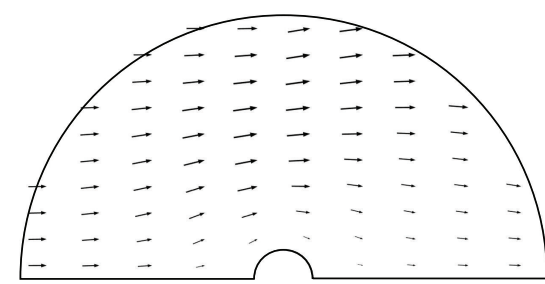

(8)

(a) $\mathrm{Re}_{1}=0.396$ and $\mathrm{St}=0.00571(f=50 \mathrm{~Hz})$

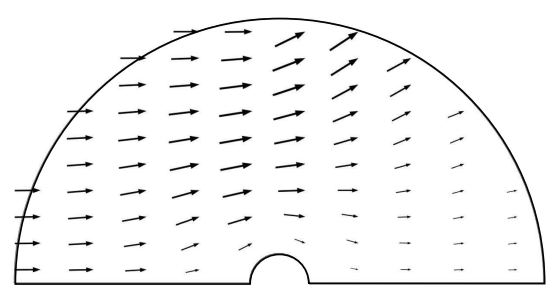

(1)

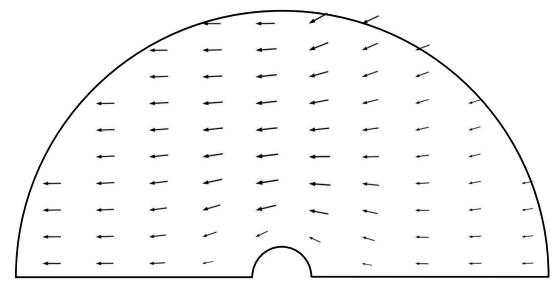

(4)

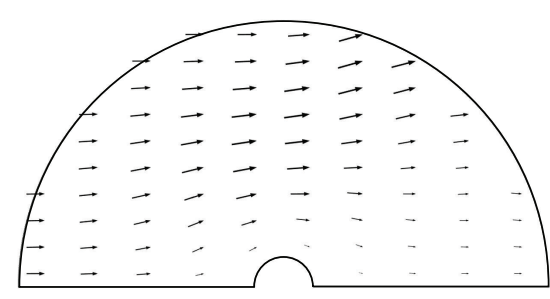

(2)

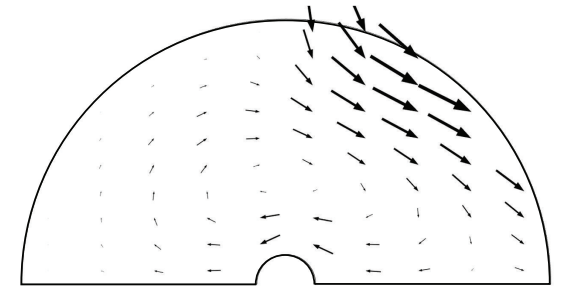

(3)

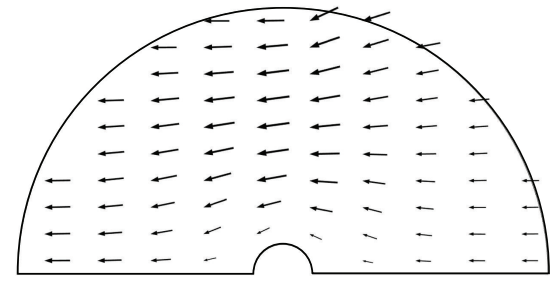

(5)

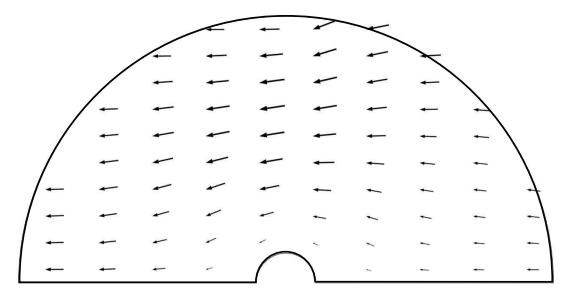

(6)

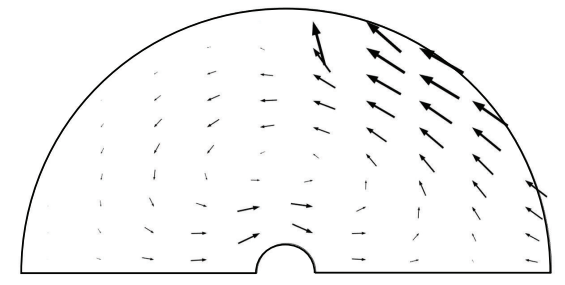

(7)

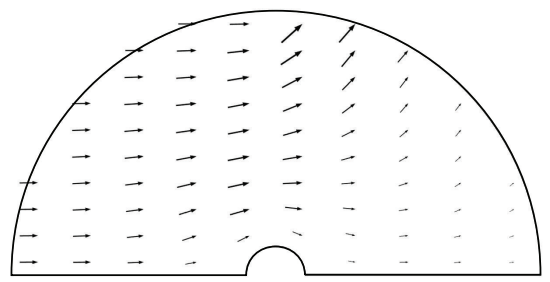

(8)

(b) $\operatorname{Re}_{1}=0.396$ and St $=0.571(f=5000 \mathrm{~Hz})$

FIGURE 5: Velocity vectors due to an acoustic field with $\mathrm{SPL}=135 \mathrm{~dB}$ around a spherical particle of diameter $100 \mu \mathrm{m}$ at different times during one cycle: (a) $\mathrm{Re}_{0}=0.0$ (no steady flow), $\mathrm{Re}_{1}=0.396$, and $\mathrm{St}=0.00571\left(50 \mathrm{~Hz}\right.$ ); (b) $\operatorname{Re}_{0}=0.0$ (no steady flow), $\mathrm{Re}_{1}=0.396$, and $\mathrm{St}=0.571$ (5000 Hz). (1) $\tau=0.0$, (2) $\tau=0.125$, (3) $\tau=0.25$, (4) $\tau=0.375$, (5) $\tau=0.5$, (6) $\tau=0.625$, (7) $\tau=0.75$, and (8) $\tau=0.875$. 

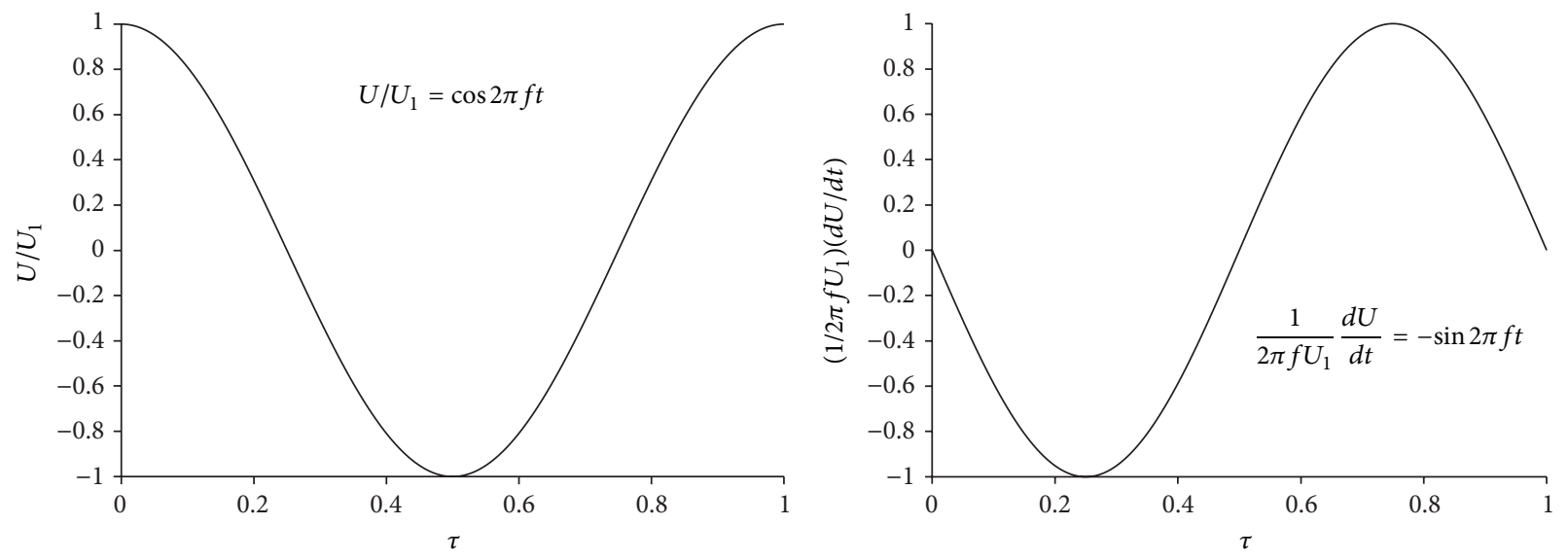

FIGURE 6: Normalized oscillating flow velocity and the flow acceleration, in the presence of an acoustic field.

TABLE 3: Summary of flow direction and axial pressure gradient for an oscillating flow.

\begin{tabular}{|c|c|c|c|}
\hline$\tau$ & Flow direction & $\begin{array}{l}\text { Angular } \\
\text { direction }\end{array}$ & $\frac{d U}{d t}$ \\
\hline $0 \sim 0.25$ & $\begin{array}{c}\text { From left to } \\
\text { right }\end{array}$ & $\beta=\pi-\theta$ & $<0$ \\
\hline $0.25 \sim 0.5$ & $\begin{array}{c}\text { From right to } \\
\text { left }\end{array}$ & $\beta=\theta$ & $<0$ \\
\hline $0.5 \sim 0.75$ & $\begin{array}{c}\text { From right to } \\
\text { left }\end{array}$ & $\beta=\theta$ & $>0$ \\
\hline $0.75 \sim 1$ & $\begin{array}{c}\text { From left to } \\
\text { right }\end{array}$ & $\beta=\pi-\theta$ & $>0$ \\
\hline$\tau$ & $\left(\frac{1}{r} \frac{\partial P}{\partial \theta}\right)_{\infty}$ & $\left(\frac{1}{r} \frac{\partial P}{\partial \theta}\right)_{w}>0$ & $\left(\frac{1}{r} \frac{\partial P}{\partial \theta}\right)_{w}<0$ \\
\hline $0 \sim 0.25$ & APG & FPG & APG \\
\hline $0.25 \sim 0.5$ & FPG & APG & FPG \\
\hline $0.5 \sim 0.75$ & APG & APG & FPG \\
\hline $0.75 \sim 1$ & FPG & FPG & APG \\
\hline
\end{tabular}

Table 3 shows a summary on the flow direction, acceleration, and pressure gradient at the infinity due to the imposed acoustic field, where $((1 / r)(\partial P / \partial \theta))_{w}$ is the axial pressure gradient on the particle surface. APG is the adverse pressure gradient, and FPG is the favorable pressure gradient.

The axial pressure gradient as well as the shear stress on the particle surface for SPL $=135 \mathrm{~dB}\left(\mathrm{Re}_{1}=0.396\right)$ and frequency $f=50 \mathrm{~Hz}(\mathrm{St}=0.00571), 5000 \mathrm{~Hz}(\mathrm{St}=0.571)$ is shown in Figure 7 during the half cycle $(\tau=0.0 \sim 0.5)$.

At $\tau=0.0$, the axial pressure gradient is 0.0 for both situations with the frequency of $50 \mathrm{~Hz}$ and $5000 \mathrm{~Hz}$ since the flow acceleration is 0.0 in the potential flow region, as given by (10) and Table 3. Thus, the curvature and the magnitude of the oscillating flow velocity $(U=0.875 \mathrm{~m} / \mathrm{s})$ are the major factors influencing the axial pressure gradient on the sphere surface. The adverse pressure gradient on the sphere surface in the downstream region is not strong enough for flow separation and forming a wake. The value of shear stress on the sphere surface is negative in the streamwise direction for both situations of the frequency of $50 \mathrm{~Hz}$ and $5000 \mathrm{~Hz}$, as shown in Figures 7(c) and 7(d).

At $\tau=0.125$, the axial pressure gradient given by (10) has positive values resulting in an adverse pressure gradient in the streamwise direction since the flow acceleration is no longer zero. At $50 \mathrm{~Hz}$, the axial pressure gradient given by (13) in the potential flow region has the maximum value of $46.6 \mathrm{~N} / \mathrm{m}^{3}$ when $\beta=\pi / 2$. Similarly, at $5000 \mathrm{~Hz}$, the axial pressure gradient has the maximum value of $4662.7 \mathrm{~N} / \mathrm{m}^{3}$. At the low frequency of $50 \mathrm{~Hz}$, the axial pressure gradient (varying in the range $0 \sim 46.6 \mathrm{~N} / \mathrm{m}^{3}$ ) is relatively small and has no effect on the axial pressure gradient on the sphere surface. Thus, the axial pressure gradient still depends mainly on curvature and the magnitude of the flow velocity $(U=0.619 \mathrm{~m} / \mathrm{s})$. When compared to the flow field at $\tau=0.0$, the flow velocity decreases from $0.875 \mathrm{~m} / \mathrm{s}$ to $0.619 \mathrm{~m} / \mathrm{s}$, resulting in a lower axial pressure gradient and shear stress on the sphere surface which can be observed in Figures 7(a) and 7(c). However, if the frequency increases to $5000 \mathrm{~Hz}$, the adverse pressure gradient (varying in the range $0 \sim 4662.7 \mathrm{~N} / \mathrm{m}^{3}$ ) at infinity is 100 times as large as that at $50 \mathrm{~Hz}$ and it is large enough to affect the axial pressure gradient on the surface. As shown in Figure 7(b), the region of the adverse pressure gradient is larger than that at $\tau=0.0$ resulting in flow separation even though the flow velocity decreases. So it can be inferred that the adverse pressure gradient on the surface is not only affected by the magnitude of flow velocity $(U=0.619 \mathrm{~m} / \mathrm{s})$ but also by the flow acceleration.

In order to illustrate the effect of flow acceleration on the sphere surface at high frequency, the axial pressure gradient and shear stress on the surface during $\tau=0.125 \sim 0.25$ are investigated, as shown in Figure 8 . At $5000 \mathrm{~Hz}$, during $\tau=$ $0.125 \sim 0.25$, the flow velocity $U$ decreases whereas the magnitude of the flow acceleration increases. Therefore, it can be concluded that the effect of curvature and the magnitude of the flow velocity will decrease; meanwhile the effect of flow acceleration on the axial pressure gradient and the shear stress on the sphere surface will increase at $5000 \mathrm{~Hz}$. However, the flow acceleration at $50 \mathrm{~Hz}$ is too small to affect the axial pressure gradient on the sphere surface when compared to 


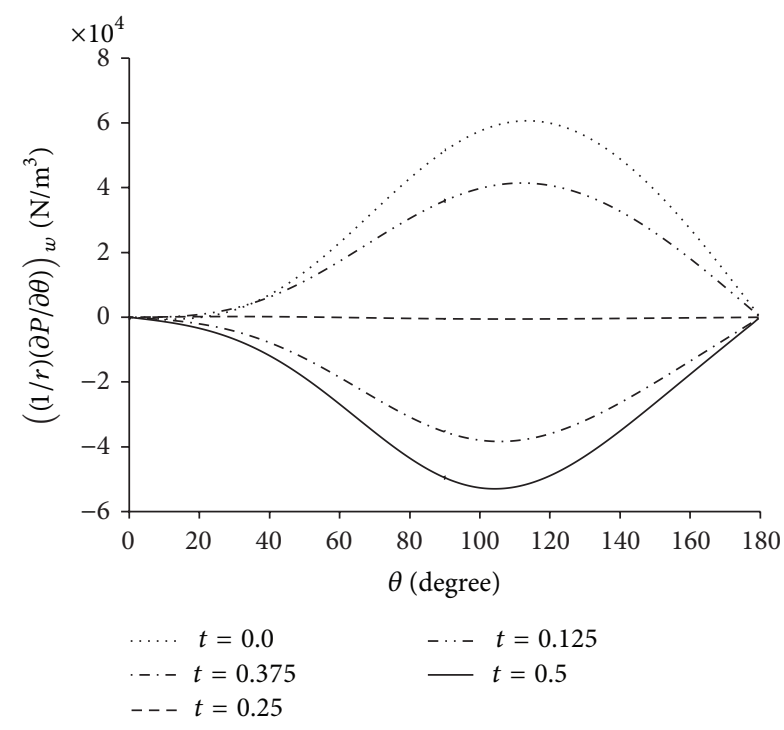

(a) Axial pressure gradient at $50 \mathrm{~Hz}$

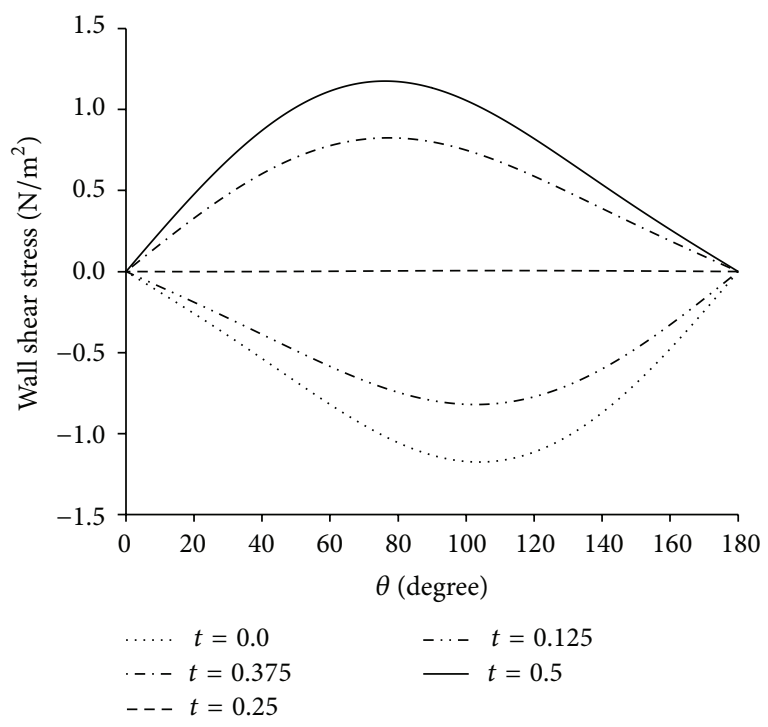

(c) Wall shear stress at $50 \mathrm{~Hz}$

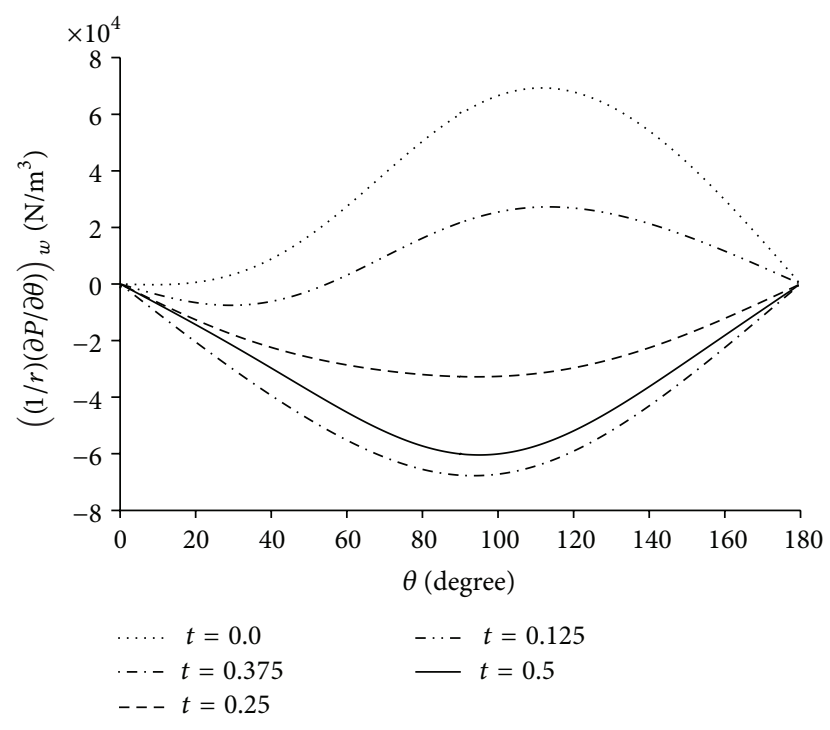

(b) Axial pressure gradient at $5000 \mathrm{~Hz}$

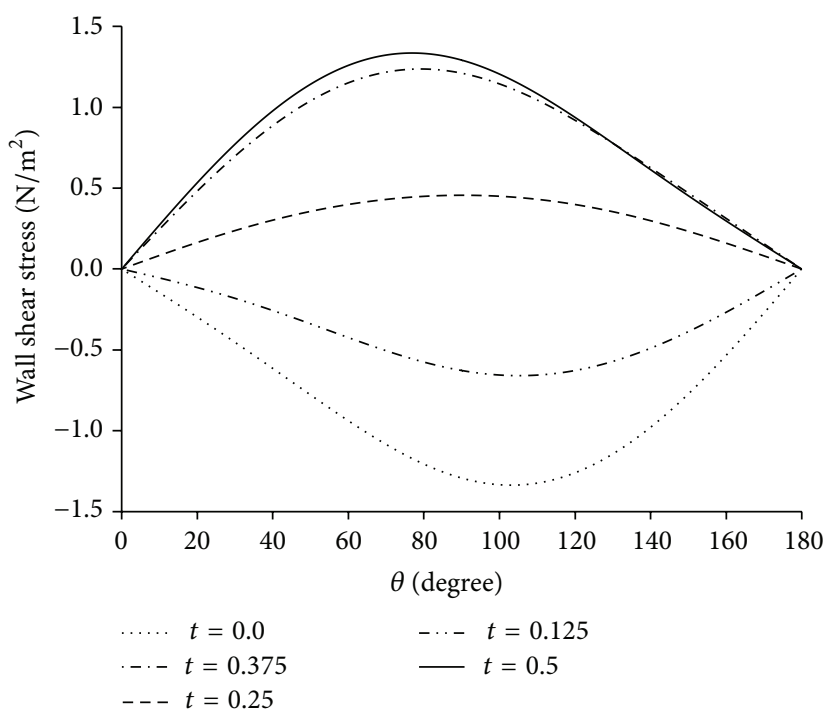

(d) Wall shear stress at $5000 \mathrm{~Hz}$

FIGURE 7: Axial pressure gradient and shear stress on the surface during the half cycle $(\tau=0.0 \sim 0.5)$ for frequency of $50 \mathrm{~Hz}(\mathrm{St}=0.00571)$ and $5000 \mathrm{~Hz}(\mathrm{St}=0.571): \mathrm{Re}_{0}=0.0$ (no steady flow), $\mathrm{Re}_{1}=0.396(\mathrm{SPL}=135 \mathrm{~dB})$.

the value at $5000 \mathrm{~Hz}$. As shown in Figures 8(a) and 8(c), the axial pressure gradient and shear stress on the sphere surface both decrease with decreasing values of the oscillating flow velocity $U$ with no flow separation at $50 \mathrm{~Hz}$. However, the flow acceleration expands the region of the adverse pressure gradient on the sphere surface resulting in a larger wake at $5000 \mathrm{~Hz}$, even though the flow velocity decreases from $0.619 \mathrm{~m} / \mathrm{s}$ to $0 \mathrm{~m} / \mathrm{s}$ during $\tau=0.125 \sim 0.25$, as shown in Figure 8(b). It can be also observed that the separation point moves upstream $\left(\beta_{s}=180^{\circ} \rightarrow 0^{\circ}\right)$, as shown in Figure 8(d).

During $\tau=0.25 \sim 0.5$, the axial pressure gradient and shear stress on the sphere surface are mainly affected by the curvature and the magnitude of the flow velocity $U$ at $50 \mathrm{~Hz}$, and the effect of the flow acceleration is negligible. However, at $5000 \mathrm{~Hz}$, the flow acceleration reaches the maximum at $\tau=0.25$, as shown in Figure 7, even though the flow velocity is zero. A flow is formed along the sphere surface and the direction is the same as the oscillating flow during $\tau=0.25 \sim$ 0.5 , as shown in Figure 5(b). This newly formed flow increases the flow velocity compared to the values at $50 \mathrm{~Hz}$.

At $\tau=0.375$, the axial pressure gradient on the sphere surface at $5000 \mathrm{~Hz}$ is favorable (negative value) due to the combined effect of the increased flow velocity and the greater flow acceleration with larger values, when compared to the values at $50 \mathrm{~Hz}$. It results in no flow separation, even though the absolute magnitude of the flow velocity is the same as that at $\tau=0.125(U=0.619 \mathrm{~m} / \mathrm{s})$, as shown in Figure $7(\mathrm{~d})$. The shear stress at $5000 \mathrm{~Hz}$ is larger than that at $50 \mathrm{~Hz}$ because of the increased axial pressure gradient, as shown in Figures 7(c) and $7(d)$. 


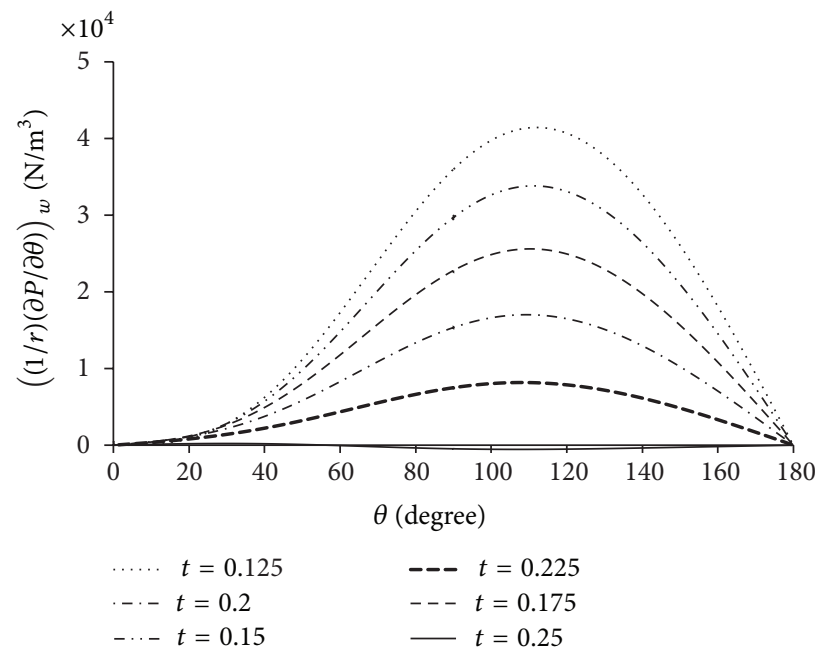

(a) Axial pressure gradient at $50 \mathrm{~Hz}$

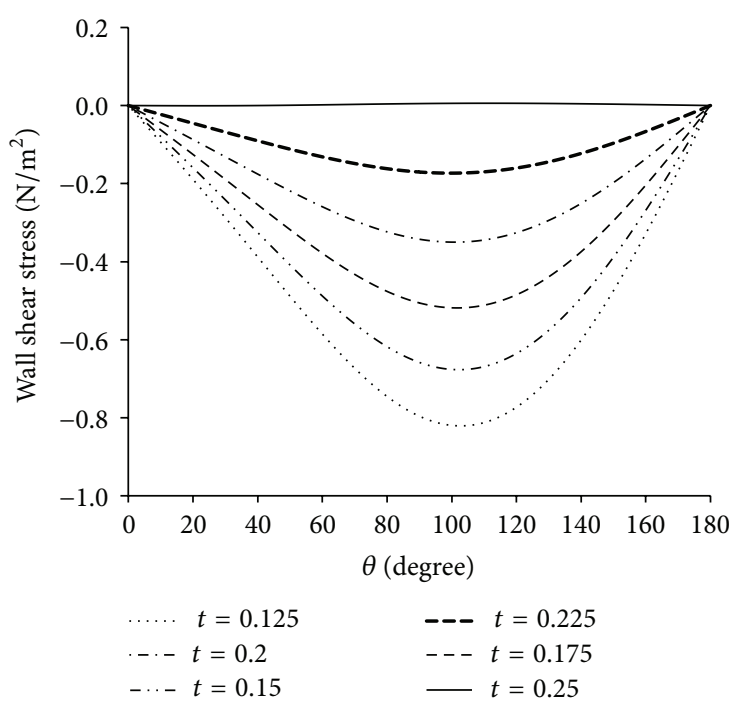

(c) Wall shear stress at $50 \mathrm{~Hz}$

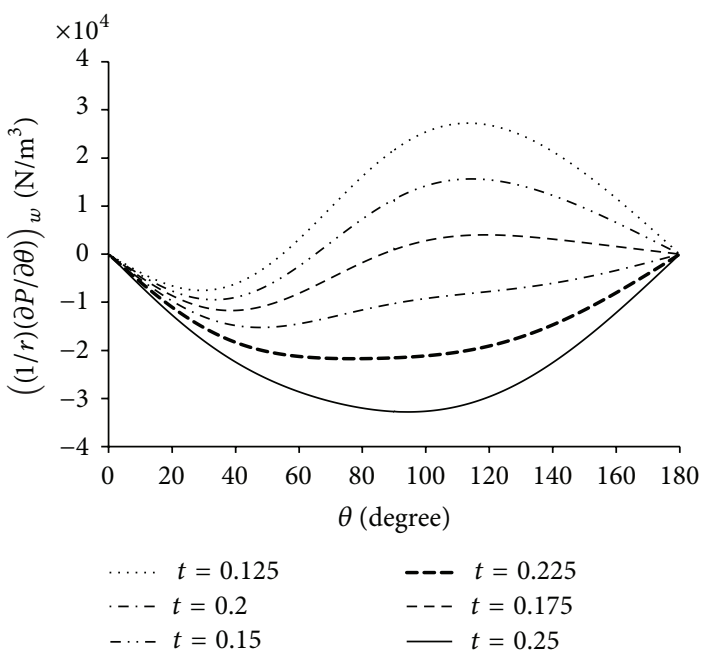

(b) Axial pressure gradient at $5000 \mathrm{~Hz}$

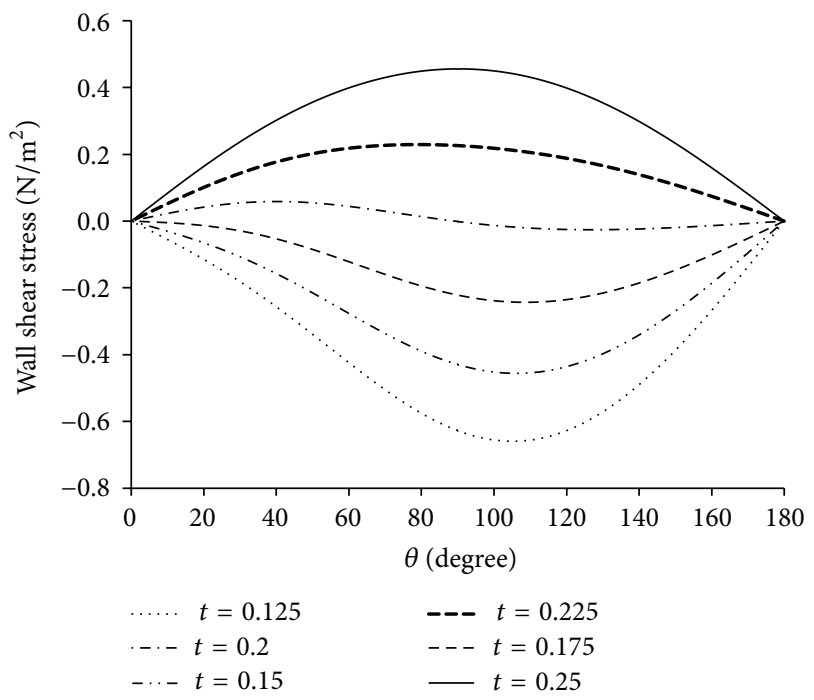

(d) Wall shear stress at $5000 \mathrm{~Hz}$

FIgure 8: Axial pressure gradient and shear stress on the surface during $\tau=0.125 \sim 0.25$ for frequencies of $50 \mathrm{~Hz}(\mathrm{St}=0.00571)$ and $5000 \mathrm{~Hz}$ $(\mathrm{St}=0.571): \mathrm{Re}_{0}=0.0$ (no steady flow), $\mathrm{Re}_{1}=0.396(\mathrm{SPL}=135 \mathrm{~dB})$.

At $\tau=0.5$, the flow acceleration in the potential flow region is zero while the flow velocity $U$ is at its maximum value of $0.875 \mathrm{~m} / \mathrm{s}$. In this case, the axial pressure gradient and shear stress are mainly affected by the curvature and flow velocity. As shown in Figures 7(a) and 7(b), the favorable pressure gradient has larger magnitude at this time compared to the values at $50 \mathrm{~Hz}$ due to the effect of the newly formed flow during $\tau=0 \sim 0.25$.

The flow velocity and acceleration of the oscillating flow in the potential flow region during $\tau=0.5 \sim 0.75$ and $\tau=$ $0.75 \sim 1.0$ have the same magnitude as those during $\tau=0 \sim 0.25$ and $\tau=0.25 \sim 0.5$, respectively, but with the opposite flow direction. Since the flow field around the spherical particle is mainly affected by the magnitude of the flow velocity and the flow acceleration, it can be inferred that the time history of axial pressure gradient and shear stress on the sphere surface during $\tau=0.5 \sim 0.75$ and $\tau=0.75 \sim 1.0$ are similar to those during $\tau=0.0 \sim 0.25$ and $\tau=0.25 \sim 0.5$, respectively, except for that they are antisymmetric.

Regarding the situation in boiler, when SPL $=135 \mathrm{~dB}$, the acoustic Reynolds number $\operatorname{Re}_{1}=0.396$, and frequency $f=$ $50 \mathrm{~Hz}$, the axial pressure gradient and the shear stress on the coal particle surface are mainly affected by the absolute magnitude of flow velocity and the curvature. No flow separation could be observed in the flow field. However, if the frequency increases to $5000 \mathrm{~Hz}$, due to the enhanced flow acceleration, flow separation could be observed, even though the acoustic Reynolds number is less than 20. The distribution of the separation point $\beta$ along the one cycle is shown in Figure 9, when acoustic Reynolds number is 0.396 and frequency is $5000 \mathrm{~Hz}$. The separation point is formed upstream along the particle surface during $\tau=0 \sim 0.25$ and 


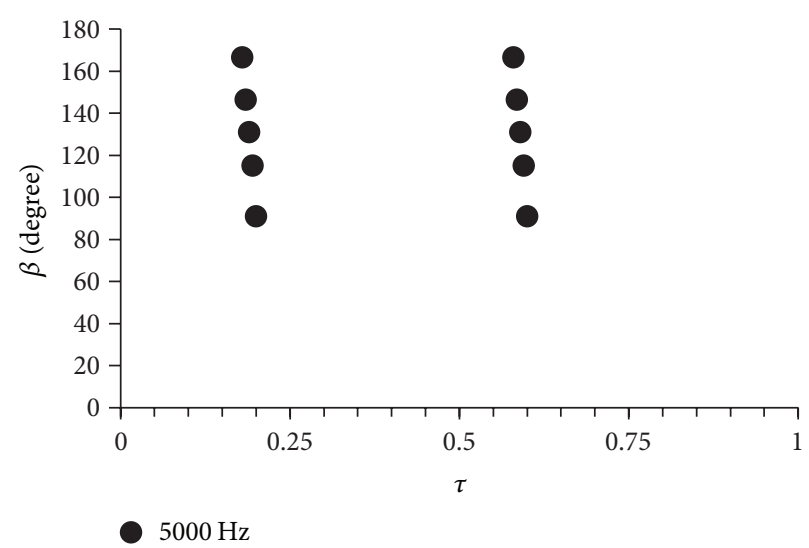

FIGURE 9: Variation of the separation angle $\beta$ as a function of $\tau$ : $\operatorname{Re}_{0}=0.0$ (no steady flow), $\operatorname{Re}_{1}=0.396$ (SPL $=135 \mathrm{~dB}$ ), and $\mathrm{St}=0.571$ $(5000 \mathrm{~Hz})$.

$\tau=0.5 \sim 0.75$, resulting in formation of a new flow which has the same direction as the flow velocity in the following time interval of $\tau=0.25 \sim 0.5$ and $\tau=0.75 \sim 1.0$, respectively.

\subsection{Flow Characteristics Induced by Acoustic Field with $S P L=$} $167 \mathrm{~dB}$. The oscillating flow induced by an acoustic field with SPL $=167 \mathrm{~dB}$ around coal particles is discussed in the following paragraph.

Figure 10 shows the velocity vectors in the simulation domain for $\operatorname{Re}_{0}=0.0$ and $\operatorname{Re}_{1}=15.75\left(U_{1}=34.8 \mathrm{~m} / \mathrm{s}, \mathrm{SPL}=\right.$ $167 \mathrm{~dB})$ when the frequency is $50 \mathrm{~Hz}(\mathrm{St}=0.000144)$ and $5000 \mathrm{~Hz}(\mathrm{St}=0.0144)$. In the potential flow region, during one cycle, the direction of the flow velocity, flow acceleration, and axial pressure gradient for $\mathrm{Re}_{1}=15.75$ are similar to the case when $\operatorname{Re}_{1}=0.396$. However, as the case of $\operatorname{Re}_{1}=15.75$ is much more larger compared to the case of $\mathrm{Re}_{1}=0.396$, even though $\mathrm{Re}_{1}$ is still less than 20 , a wake is formed at both frequency of $50 \mathrm{~Hz}$ and $5000 \mathrm{~Hz}$, as shown in Figures 10(a) and 10(b). It can also be observed that the wake size at $5000 \mathrm{~Hz}$ is larger than that at $50 \mathrm{~Hz}$.

At $50 \mathrm{~Hz}$, the axial pressure gradient at $\mathrm{Re}_{1}=15.75$ in the potential flow is still too small to affect the axial pressure gradient on the sphere surface, which is six times as large as the value at $\operatorname{Re}_{1}=0.396$. Therefore, the flow field, separation angle, axial pressure gradient, and shear stress on the sphere surface are also affected by the curvature and flow velocity. As for the case at $5000 \mathrm{~Hz}$, flow acceleration becomes one of the most important factors, just similar to the case when $\operatorname{Re}_{1}=$ 0.396 .

Figure 11 shows the axial pressure gradient and shear stress on the particle surface for SPL $=167 \mathrm{~dB}\left(\mathrm{Re}_{1}=15.75\right)$ and $f=50 \mathrm{~Hz}(\mathrm{St}=0.000144)$ and $5000 \mathrm{~Hz}(\mathrm{St}=0.0144)$ during the half cycle $(\tau=0.0 \sim 0.5)$. Figure 12 shows the axial pressure gradient and shear stress for SPL $=167 \mathrm{~dB}\left(\mathrm{Re}_{1}=\right.$ $15.75)$ and $f=50 \mathrm{~Hz}(\mathrm{St}=0.000144)$ and $5000 \mathrm{~Hz}(\mathrm{St}=$ 0.0144 ) during $\tau=0.125 \sim 0.25$. It can be observed in Figures 11 (a) and 11 (c) that when compared to the results for $\operatorname{Re}_{1}=$ 0.396 at $50 \mathrm{~Hz}$, the magnitude of the axial pressure gradient and shear stress on the sphere surface during one cycle are much larger. This could explain the fact that the flow separation could always be observed when SPL is larger than
$162 \mathrm{~dB}$ in the range of audible frequency. The distribution of the axial pressure gradient and shear stress on the sphere surface at $\operatorname{Re}_{1}=15.75$ are similar to those at $\operatorname{Re}_{1}=0.396$. During $\tau=0.0 \sim 0.25$, due to the fact that the flow acceleration reaches its minimum value at $\tau=0.0$ and the maximum value at $\tau=0.25$, an adverse pressure gradient is formed in the potential flow region. Therefore, at $f=5000 \mathrm{~Hz}$, the favorable pressure gradient in the upstream region is decreasing; meanwhile the adverse pressure gradient in the wake is increasing at $\tau=0.125$ and $\tau=0.25$ because of the increasing flow acceleration. The magnitude of the shear stress on the sphere surface is proportional to the magnitude of the axial pressure gradient on the surface, as shown in Figures 11(c) and 11(d).

Similar to the case of $\operatorname{Re}_{1}=0.396$ and $f=5000 \mathrm{~Hz}$, even though the flow velocity $U$ is zero, a flow is formed along with the direction from $\theta=0^{\circ}$ to $180^{\circ}$ for $\operatorname{Re}_{1}=15.75$ and $f=5000 \mathrm{~Hz}$, as shown in Figure 10(b). The magnitude of its velocity increases with increasing $\mathrm{Re}_{1}$. As shown in Figure 11(b), the magnitude of the axial pressure gradient on the sphere surface during $\tau=0.25 \sim 0.5$ increases, due to the combined effect of the newly formed flow and the acceleration of the oscillating flow. The axial pressure gradient and shear stress on the sphere surface during $\tau=0.5 \sim 0.75$ and $\tau=0.75 \sim 1.0$ are very similar to those during $\tau=0.0 \sim$ 0.25 and $\tau=0.25 \sim 0.5$, respectively, except for that they are antisymmetric which have been discussed previously.

Figure 13 shows the distribution of the separation point $\beta$ along one cycle for $\operatorname{Re}_{1}=15.75$. It can be found that the separation point $\beta$ at $50 \mathrm{~Hz}$ moves downstream from $0^{\circ}$ to $180^{\circ}$ with decreasing magnitude of the oscillating velocity, resulting in the decreasing wake size during $\tau=$ $0.0 \sim 0.25$ and $\tau=0.5 \sim 0.75$. However, the separation point at $5000 \mathrm{~Hz}$ moves upstream from $180^{\circ}$ to $0^{\circ}$ resulting in the increasing wake size, due to the increasing effects of flow acceleration (adverse pressure gradient). During $\tau=0.25 \sim$ 0.5 and $\tau=0.75 \sim 1.0$, the separation point at $50 \mathrm{~Hz}$ moves upstream with increased spread. However, at $5000 \mathrm{~Hz}$, the formation of the flow separation is later than that at $50 \mathrm{~Hz}$ resulting in the larger values of separation angle $\beta$ and smaller wake size. 


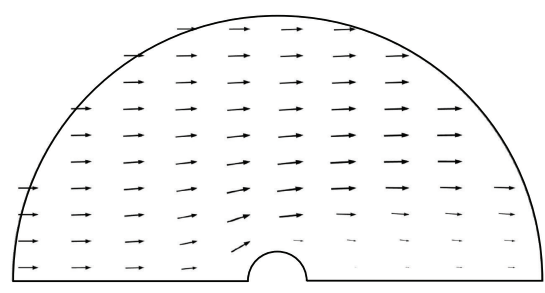

(1)

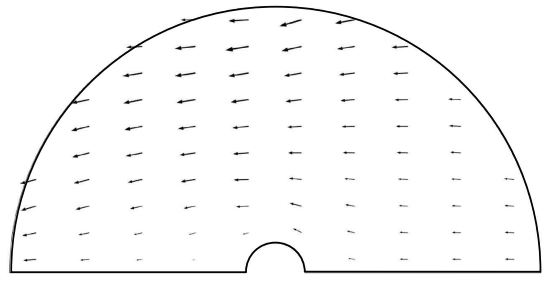

(4)

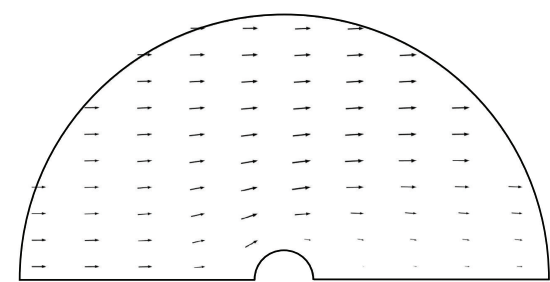

(2)

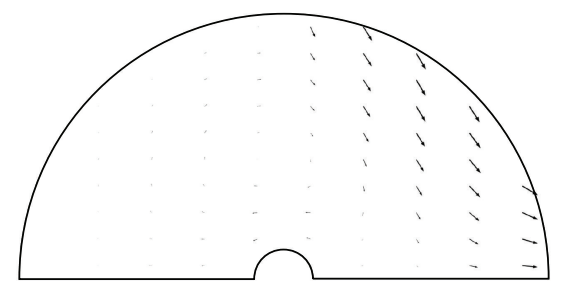

(3)

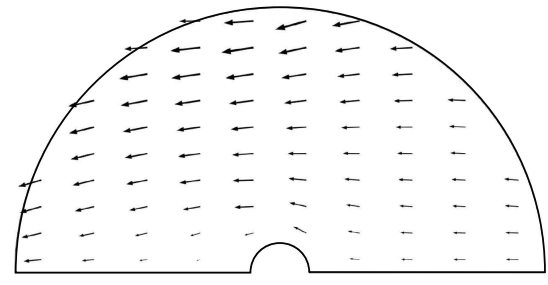

(5)

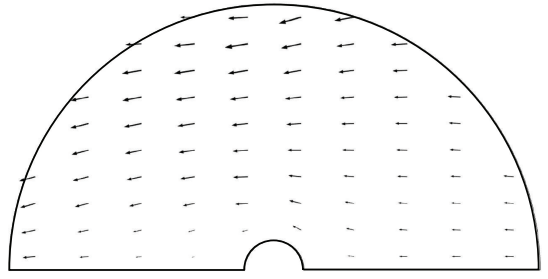

(6)

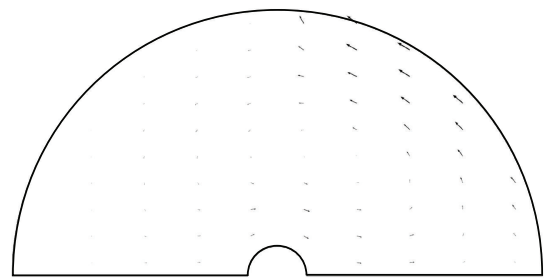

(7)

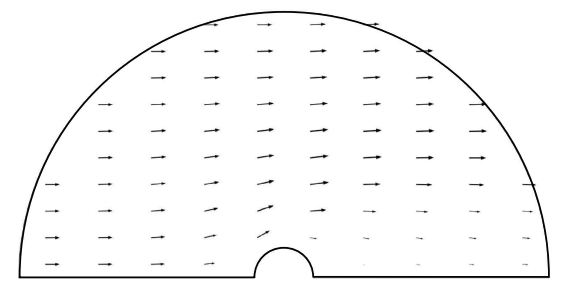

(8)

(a) $\mathrm{Re}_{1}=15.75$ and $\mathrm{St}=0.000144(f=50 \mathrm{~Hz})$

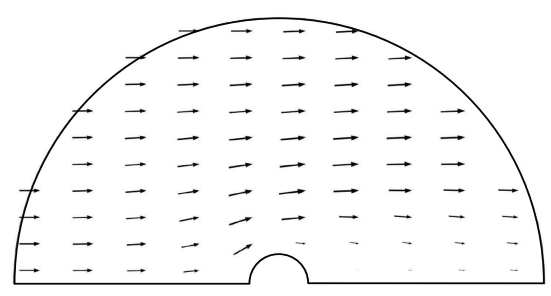

(1)

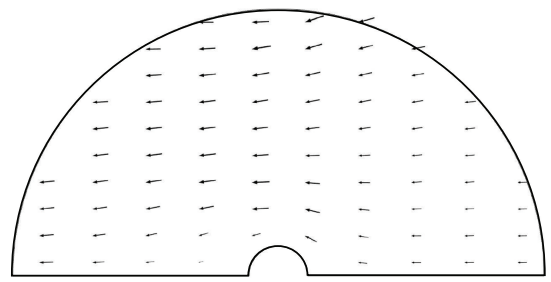

(4)

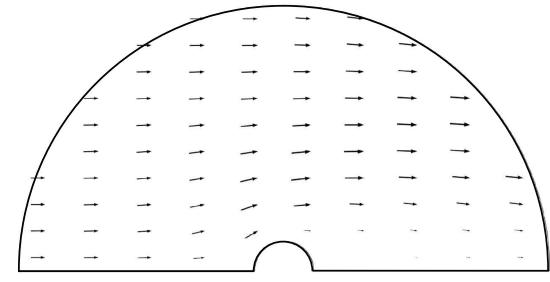

(2)

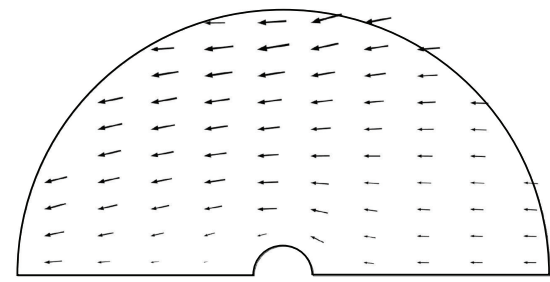

(5)

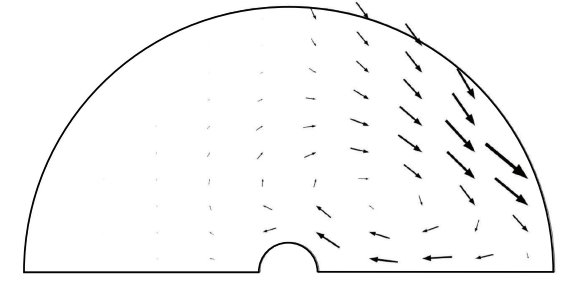

(3)

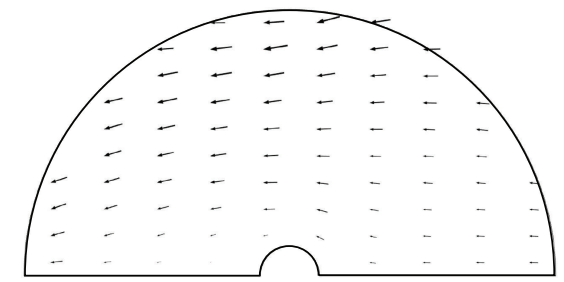

(6)

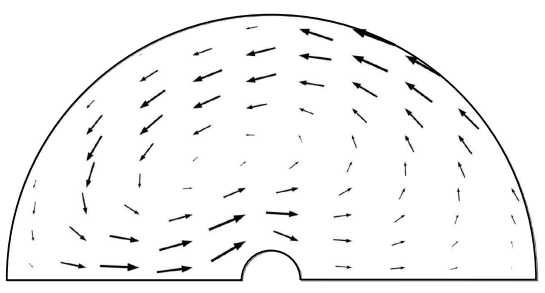

(7)

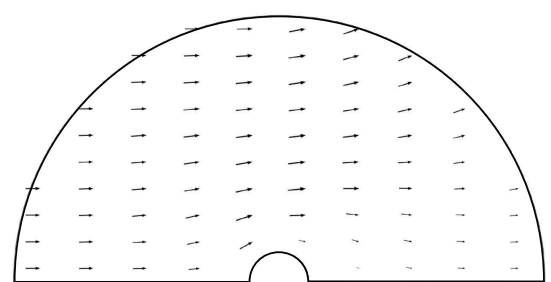

(8)

(b) $\mathrm{Re}_{1}=15.75$ and $\mathrm{St}=0.0144(f=5000 \mathrm{~Hz})$

FIGURE 10: Velocity vectors due to an acoustic field with SPL $=167 \mathrm{~dB}$ around a spherical particle of diameter $100 \mu \mathrm{m}$ at different times during one cycle: (a) $\operatorname{Re}_{0}=0.0$ (no steady flow), $\operatorname{Re}_{1}=15.75$, and $\mathrm{St}=0.000144(50 \mathrm{~Hz}) ;(\mathrm{b}) \mathrm{Re}_{0}=0.0$ (no steady flow), $\mathrm{Re}_{1}=15.75$, and $\mathrm{St}=0.0144$ (5000 Hz). (1) $\tau=0.0$, (2) $\tau=0.125$, (3) $\tau=0.25$, (4) $\tau=0.375$, (5) $\tau=0.5$, (6) $\tau=0.625$, (7) $\tau=0.75$, and (8) $\tau=0.875$. 


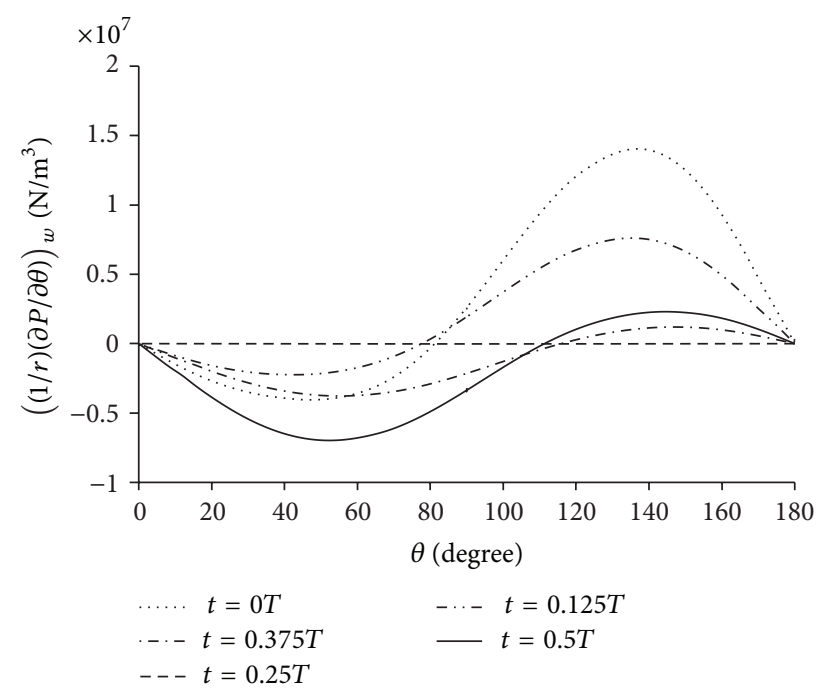

(a) Axial pressure gradient at $50 \mathrm{~Hz}$

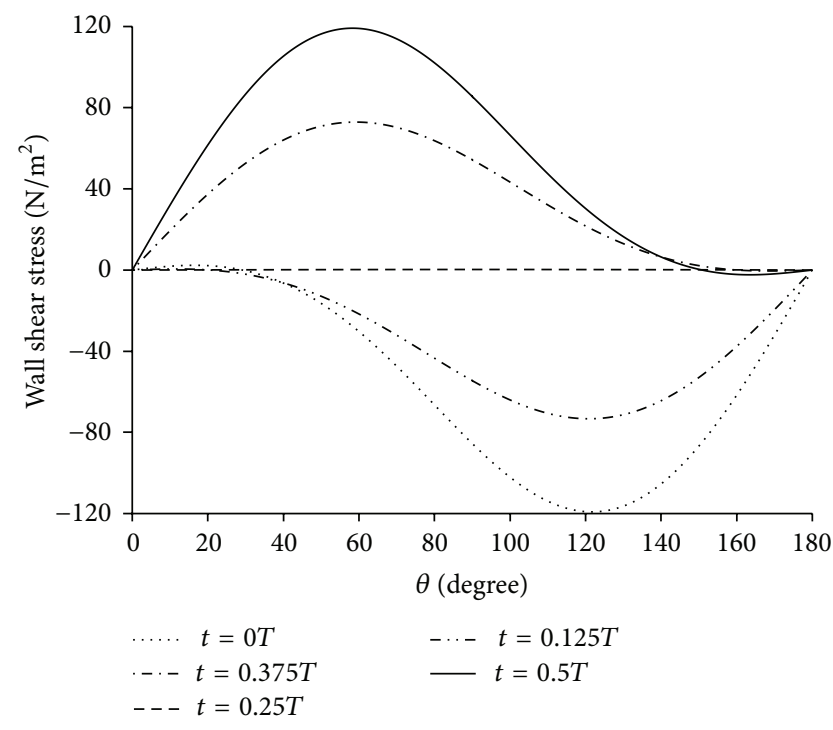

(c) Wall shear stress at $50 \mathrm{~Hz}$

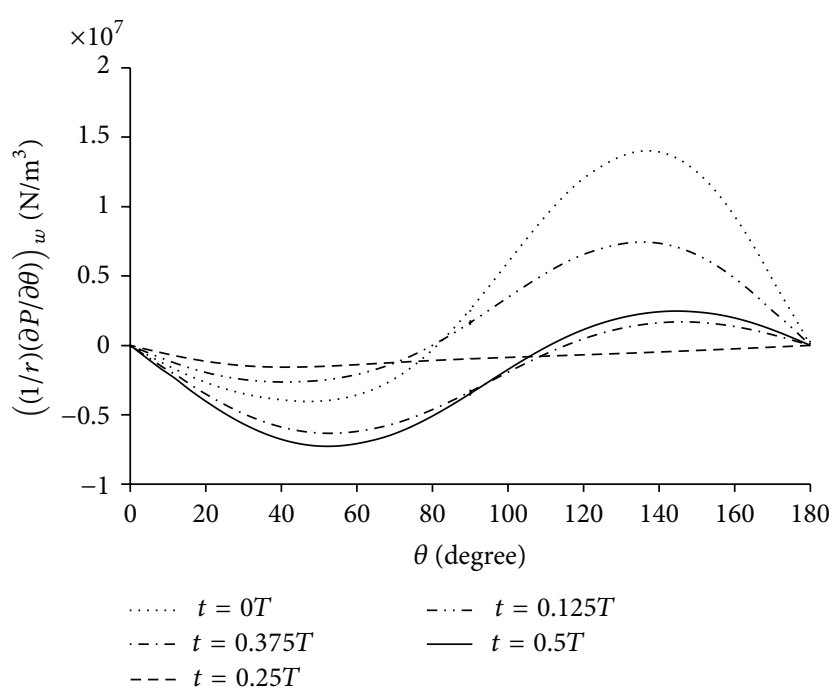

(b) Axial pressure gradient at $5000 \mathrm{~Hz}$

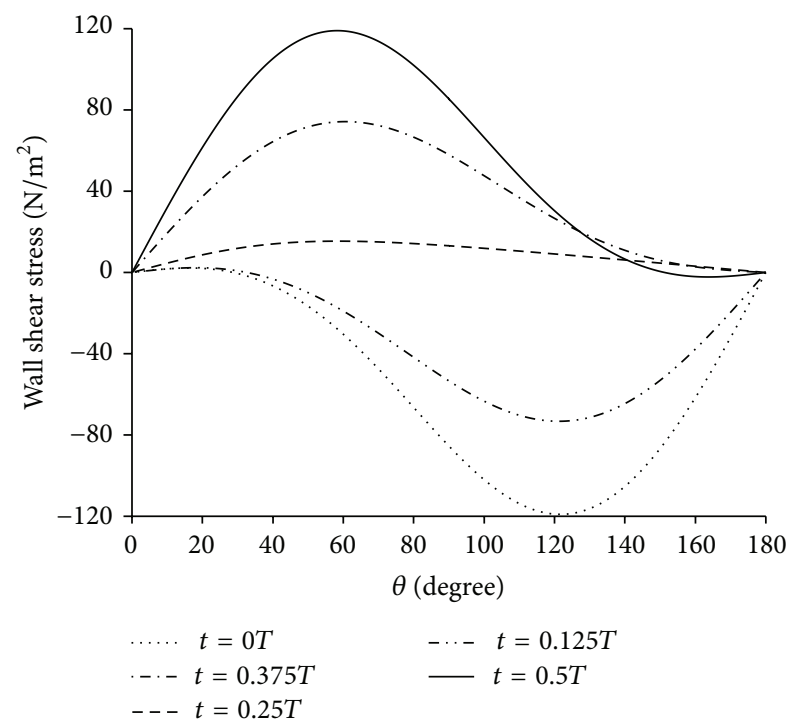

(d) Wall shear stress at $5000 \mathrm{~Hz}$

Figure 11: Axial pressure gradient and shear stress on the surface during the half cycle $(\tau=0.0 \sim 0.5)$ for frequency of $50 \mathrm{~Hz}(\mathrm{St}=0.000144)$ and $5000 \mathrm{~Hz}$ (St = 0.0144): $\operatorname{Re}_{0}=0.0$ (no steady flow), $\operatorname{Re}_{1}=15.75(\mathrm{SPL}=167 \mathrm{~dB})$.

When SPL $=167 \mathrm{~dB}$, which is large enough to be treated as the high-intensity sound. The high-intensity acoustic field would generate a steady motion (acoustic streaming) around the spherical particle, which is independent of the direction of the oscillating flow. However, the magnitude of this acoustic streaming is too small to affect the flow field [16].

It is well known that boundary layer would be formed on the surface when a viscous flow passes over an object. The flow velocity within boundary layer increases sharply from zero to the external potential flow velocity along the normal direction of the surface. If there exists temperature difference between the flow and the object, heat shall transfer through the boundary layer by heat conduction, resulting in a lower heat transfer efficiency. However, if there exists flow separation on the particle surface, the vortexes are periodically formed nearby and then taken away by the mainstream.
The flue gas in the mainstream is drawn in the boundary layer as the medium for convective heat transfer, resulting in a higher heat transfer efficiency. The local Nusselt number on the particle surface is proportional to the absolute magnitude of the wall shear stress.

In the power plant boiler, pulverized coal particles burning in the combustion zone release heat to the flue gas. No flow separation would be observed around the coal particles, when a steady flow passed over them, due to the viscosity of the flue gas and temperature in furnace. However, when an oscillating flow induced by an acoustic field is applied, flow separation would be observed on the surface. Since the vortexes are formed periodically, the flow field around particles has been enhanced, so as to the rate of heat and mass transfer from coal particles. 

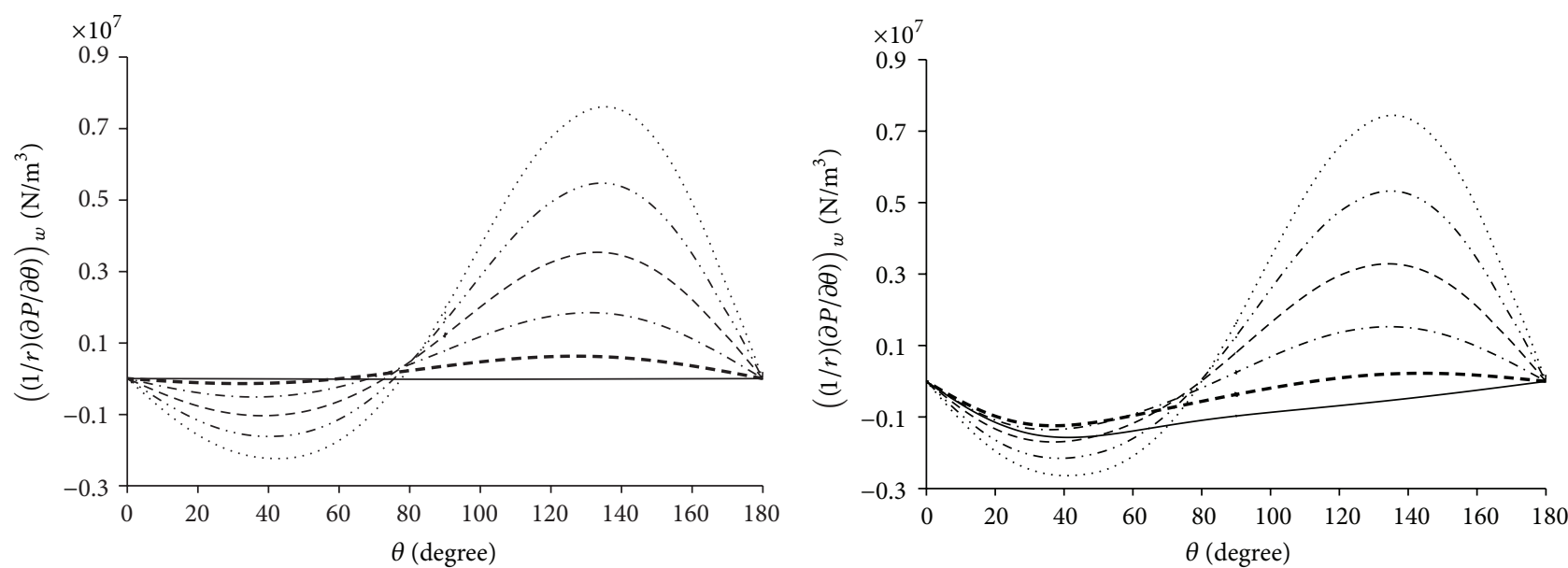

$$
\begin{array}{ll}
\cdots \cdots t=0.125 T & --t=0.225 T \\
\cdots-t=0.2 T & --t=0.175 T \\
-\cdots-t=0.15 T & -t=0.25 T
\end{array}
$$

(a) Axial pressure gradient at $50 \mathrm{~Hz}$

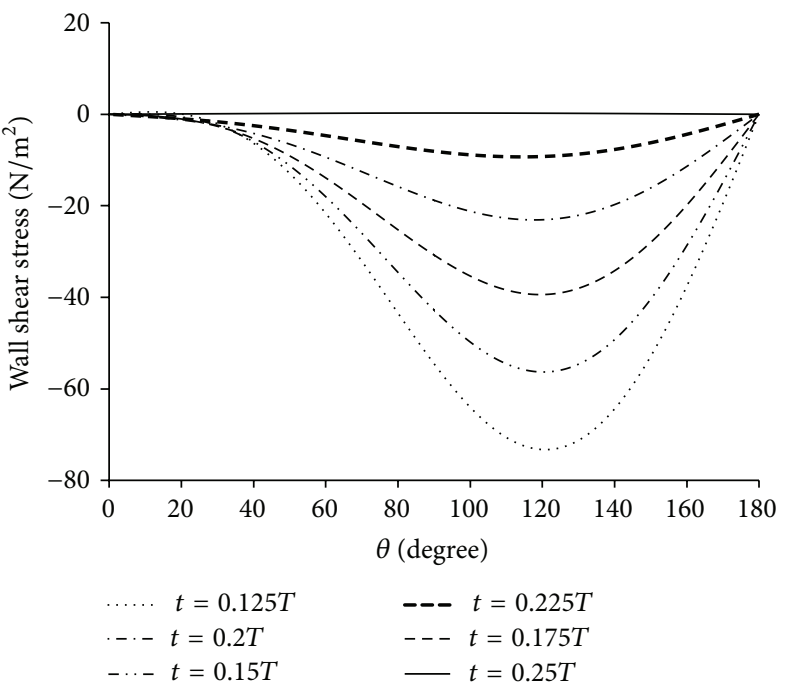

(c) Wall shear stress at $50 \mathrm{~Hz}$

$$
\begin{aligned}
\cdots \cdots & t=0.125 T & ---t & =0.225 T \\
\cdots-t & =0.2 T & ---t & =0.175 T \\
-\cdots-t & =0.15 T & - & =0.25 T
\end{aligned}
$$

(b) Axial pressure gradient at $5000 \mathrm{~Hz}$

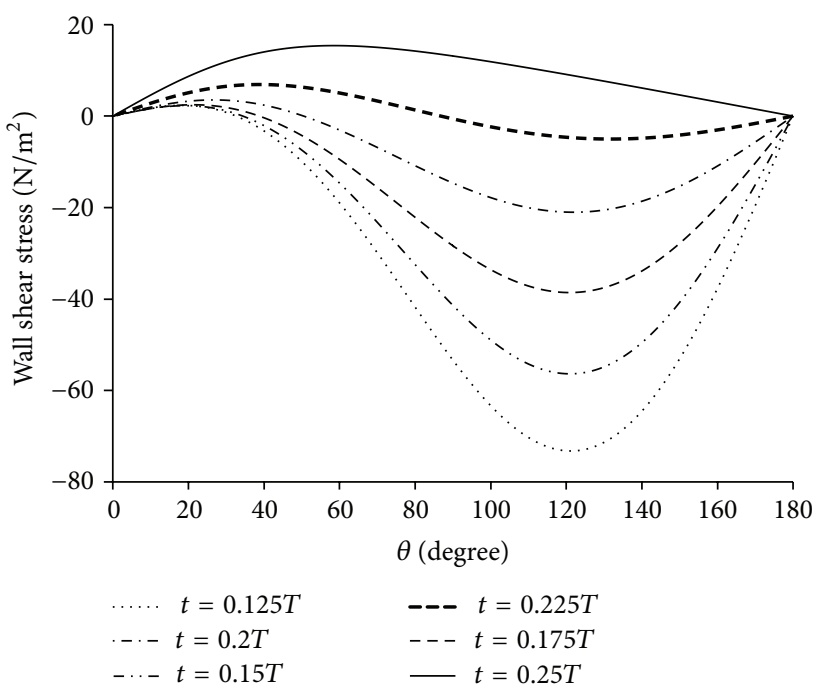

(d) Wall shear stress at $5000 \mathrm{~Hz}$

FiguRE 12: Axial pressure gradient and shear stress on the surface during $\tau=0.125 \sim 0.25$ for frequencies $50 \mathrm{~Hz}(\mathrm{St}=0.000144)$ and $5000 \mathrm{~Hz}$ $(\mathrm{St}=0.0144): \operatorname{Re}_{0}=0.0$ (no steady flow) and $\operatorname{Re}_{1}=15.75(\mathrm{SPL}=167 \mathrm{~dB})$.

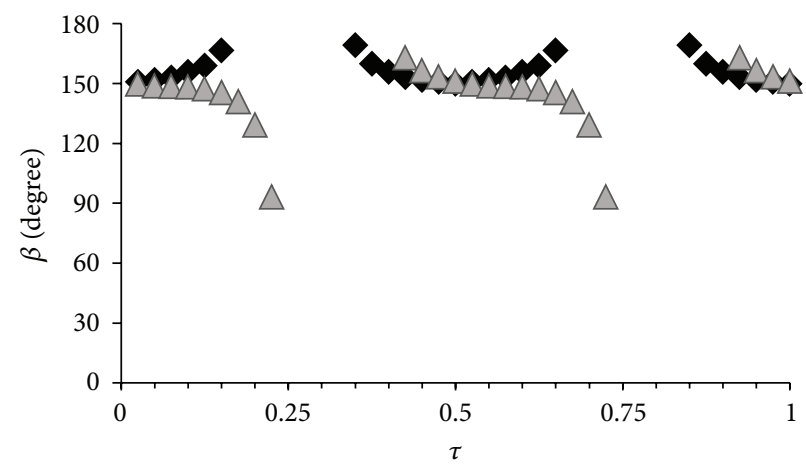

- $50 \mathrm{~Hz}$

$\triangle 5000 \mathrm{~Hz}$

FiguRE 13: Variation of the separation angle $\beta$ as a function of $\tau$ for frequencies of $50 \mathrm{~Hz}(\mathrm{St}=0.000144)$ and $5000 \mathrm{~Hz}(\mathrm{St}=0.0144): \mathrm{Re}_{0}=$ 0.0 (no steady flow) and $\operatorname{Re}_{1}=15.75(\mathrm{SPL}=167 \mathrm{~dB})$. 


\section{Conclusions}

In order to investigate oscillating flow induced by an acoustic field around coal particles in the power plant boiler, the axisymmetric, laminar momentum and mass conservation equations have been solved numerically with different acoustic Reynolds numbers and Strouhal numbers. The following can be concluded from the modelling results.

The axial pressure gradient, shear stress, and flow separation on the sphere surface all depend on the change of the flow velocity $U$ due to body curvature and flow acceleration; the result is in good accordance with Ha's work [17]. At low frequency $(\sim 50 \mathrm{~Hz})$, the curvature is the dominant factor, resulting in increasing values of these values with increasing $U$ whereas, at a high frequency $(\sim 5000 \mathrm{~Hz})$, the effect of the flow acceleration becomes more obvious; there exists the wake with increasing size even though the flow velocity decreases, due to the combined effects of the curvature and the flow acceleration.

It is also found that flow separation would be always observed on the particle surface even though the acoustic Reynolds number is less than 20, only if the sound frequency is larger than a certain value.

When an acoustic field is applied to enhance the combustion for the boiler, flow separation has an important influence on the rate of the heat and mass transfer from the coal particles. Since the rate of heat and mass transfer increases, the combustion efficiency in the power plant boiler would be enhanced. The higher the sound frequency in the range of audio frequency is and the higher the sound pressure level is, the higher the combustion efficiency would be achieved.

\section{Competing Interests}

The authors declare that they have no competing interests.

\section{Acknowledgments}

The authors acknowledge support from the National Natural Science Foundation of China (11474091 and 11274111), the Natural Science Foundation of Hebei Province of China (A2015502077), and the Fundamental Research Funds for the Central Universities (2015XS105).

\section{References}

[1] R. S. Alassar and H. M. Badr, "Oscillating viscous flow over a sphere," Computers \& Fluids, vol. 26, no. 7, pp. 661-682, 1997.

[2] P. Wolf, G. Staffelbach, L. Y. M. Gicquel, J.-D. Müller, and T. Poinsot, "Acoustic and Large Eddy Simulation studies of azimuthal modes in annular combustion chambers," Combustion and Flame, vol. 159, no. 11, pp. 3398-3413, 2012.

[3] H. Kiani, D.-W. Sun, and Z. Zhang, "Effects of processing parameters on the convective heat transfer rate during ultrasound assisted low temperature immersion treatment of a stationary sphere," Journal of Food Engineering, vol. 115, no. 3, pp. 384-390, 2013.

[4] N. P. Dhanalakshmi, R. Nagarajan, N. Sivagaminathan, and B. V. S. S. S. Prasad, "Acoustic enhancement of heat transfer in furnace tubes," Chemical Engineering and Processing: Process Intensification, vol. 59, pp. 36-42, 2012.

[5] S. Hasegawa, K. Kondo, and Y. Oshinoya, "Experimental verification of heat transport by acoustic wave," Applied Thermal Engineering, vol. 78, pp. 551-555, 2015.

[6] A. B. Bassett, A Treatise in Hydrodynamics, Deighton, 1888.

[7] F. Odar and S. Hamilton, "Force on a sphere accelerating in a viscous fluid," Journal of Fluid Mechanics, vol. 18, no. 2, pp. 302314, 1964.

[8] R. Mei, "Flow due to an oscillating sphere and an expression for unsteady drag on the sphere at finite Reynolds number," Journal of Fluid Mechanics, vol. 270, pp. 133-174, 1994.

[9] E. J. Chang and M. R. Maxey, "Unsteady flow about a sphere at low to moderate Reynolds number. Part 1. Oscillatory motion," Journal of Fluid Mechanics, vol. 277, pp. 347-379, 1994.

[10] R. S. Alassar, "Acoustic streaming on spheres," International Journal of Non-Linear Mechanics, vol. 43, no. 9, pp. 892-897, 2008.

[11] C. Pozrikidis, "A study of linearized oscillatory flow past particles by the boundary-integral method," Journal of Fluid Mechanics, vol. 202, pp. 17-41, 1989.

[12] M. Y. Ha, A throretical study of augmentation of particle combustion via aocusitc enhancement of heat and mass transfer [Ph.D. thesis], The Pennsylvania State University, Pennsylvania, $\mathrm{Pa}, \mathrm{USA}, 1990$.

[13] S. S. Sadhal, "Acoustofluidics 15: streaming with sound waves interacting with solid particles," Lab on a Chip, vol. 12, no. 15, pp. 2600-2611, 2012.

[14] S. V. Patankar, Numerical Heat Transfer and Fluid Flow, Hemisphere, Washington, DC, USA, 1980.

[15] H. Niazmand and M. Renksizbulut, "Surface effects on transient three-dimensional flows around rotating spheres at moderate Reynolds numbers," Computers \& Fluids, vol. 32, no. 10, pp. 1405-1433, 2003.

[16] M. Y. Ha and S. Yavuzkurt, "A theoretical investigation of acoustic enhancement of heat and mass transfer-II. Oscillating flow with a steady velocity component," International Journal of Heat and Mass Transfer, vol. 36, no. 8, pp. 2193-2202, 1993.

[17] M. Y. Ha, "A theoretical study on the acoustically driven oscillating flow around small spherical particle," KSME Journal, vol. 6, pp. 49-57, 1992. 


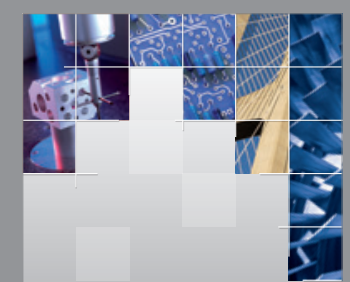

\section{Enfincering}
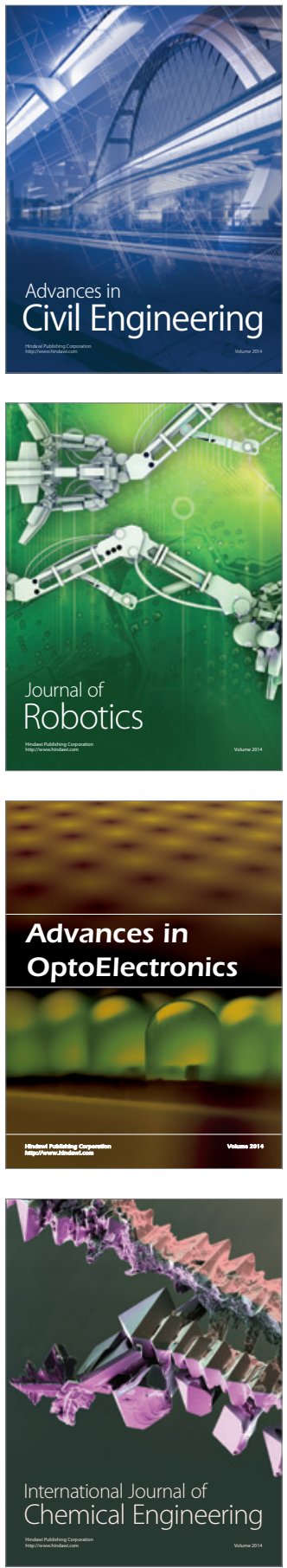

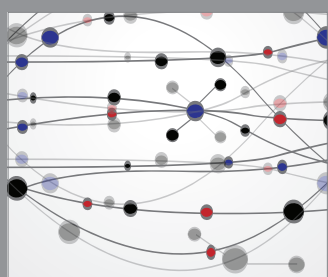

The Scientific World Journal

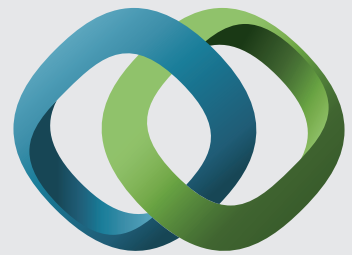

\section{Hindawi}

Submit your manuscripts at

http://www.hindawi.com
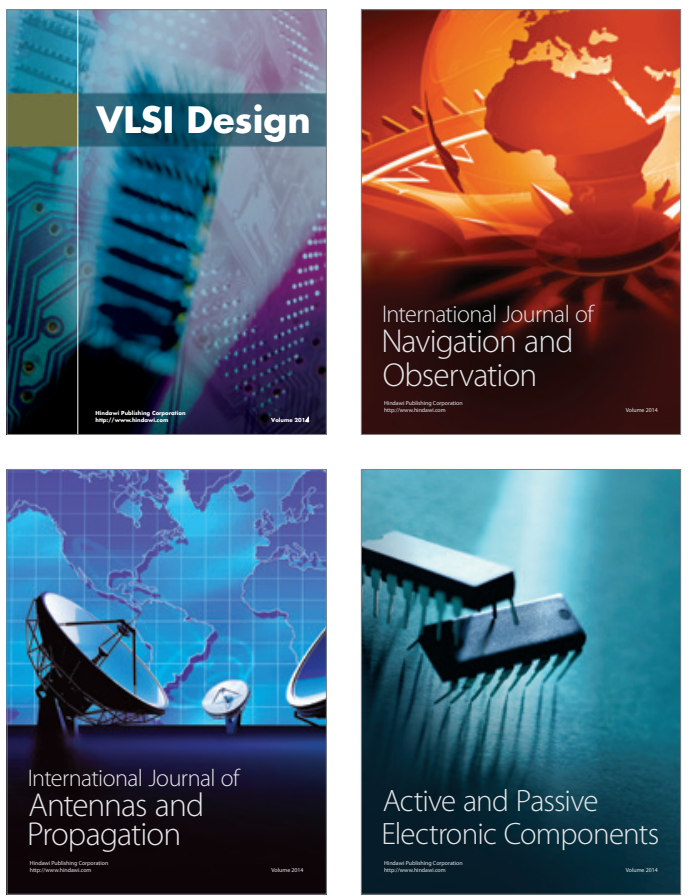
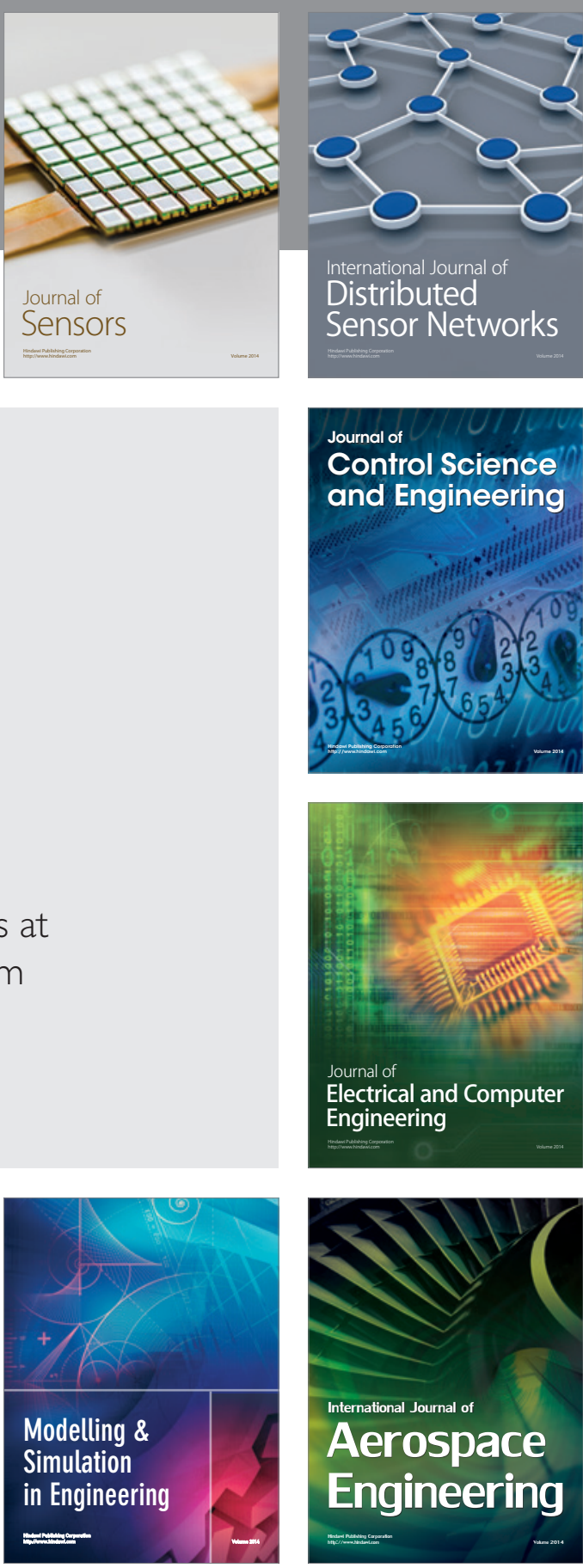

International Journal of

Distributed

Sensor Networks

Journal of

Control Science

and Engineering
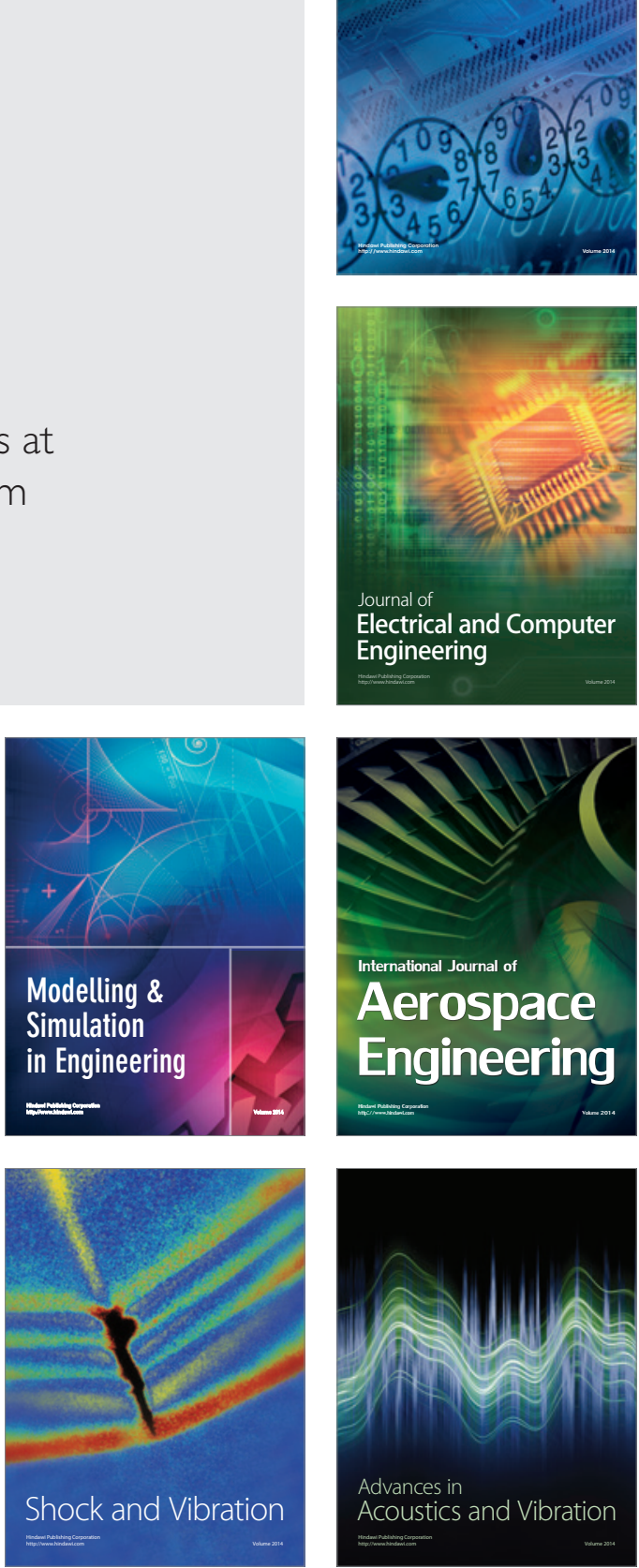